Discussion Paper No. 03-45

\title{
Multifaktormodelle zur Erklärung deutscher Aktienrenditen: Eine empirische Analyse
}

Andreas Ziegler, Elke Eberts, Michael Schröder, Anja Schulz und Richard Stehle

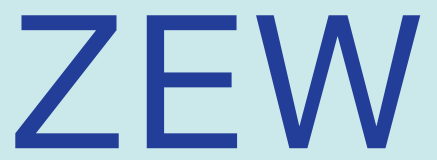

Zentrum für Europäische Wirtschaftsforschung GmbH

Centre for European Economic Research 
Discussion Paper No. 03-45

\title{
Multifaktormodelle zur Erklärung deutscher Aktienrenditen: Eine empirische Analyse
}

\author{
Andreas Ziegler, Elke Eberts, Michael Schröder, \\ Anja Schulz und Richard Stehle
}

Download this ZEW Discussion Paper from our ftp server:

ftp://ftp.zew.de/pub/zew-docs/dp/dp0345.pdf

Die Discussion Papers dienen einer möglichst schnellen Verbreitung von neueren Forschungsarbeiten des ZEW. Die Beiträge liegen in alleiniger Verantwortung der Autoren und stellen nicht notwendigerweise die Meinung des ZEW dar.

Discussion Papers are intended to make results of ZEW research promptly available to other economists in order to encourage discussion and suggestions for revisions. The authors are solely responsible for the contents which do not necessarily represent the opinion of the ZEW. 


\section{Das Wichtigste in Kürze}

Die systematische Erklärung von Aktienrenditen ist seit langem eine der zentralen Herausforderungen der finanzökonomischen Forschung. In dieser Hinsicht ist bis heute das Capital Asset Pricing Model (CAPM) der verbreitetste Ansatz. Entsprechend dem CAPM reicht der so genannte "Beta-Faktor" aus, um die erwartete Aktienrendite zu erklären. Allerdings hat eine Vielzahl empirischer Studien schon ab Anfang der 1980er Jahre gezeigt, dass auch andere Faktoren einen Beitrag zur Erklärung von Aktienrenditen liefern können. Da Einflüsse solcher Faktoren nicht durch das CAPM erfasst werden, werden sie in der finanzökonomischen Literatur häufig als Anomalien bezeichnet. Insbesondere Untersuchungen von Fama/French $(1992,1993)$ haben jedoch in der jüngeren Vergangenheit die Diskussion um ein zum CAPM alternatives Kapitalmarktmodell verstärkt. Daran anknüpfend wird in diesem Papier untersucht, ob Multifaktormodelle nach Fama/French (1993) am deutschen Aktienmarkt analog zum US-amerikanischen, kanadischen und britischen Aktienmarkt eine höhere Erklärungskraft für die Überschussrendite von Aktienportfolios über den risikolosen Zins aufweisen als das CAPM.

Dazu werden Portfolios auf der Grundlage des Marktwertes sowie des Quotienten aus Buchund Marktwert der betrachteten im amtlichen Handel an der Frankfurter Wertpapierbörse notierten Industrieunternehmen gebildet. Neben der Überschussrendite des Aktienmarktes werden zur Erklärung der Überschussrendite dieser Aktienportfolios zwei weitere Risikofaktoren des Aktienmarktes (die sich aus dem Marktwert sowie dem Quotienten aus Buch- und Marktwert ableiten) sowie zwei zusätzliche Risikofaktoren des Anleihenmarktes (die sich aus der Zinsstruktur und dem Ausfallrisiko ableiten) in die Betrachtung einbezogen. Die Analyse selbst erfolgt in zwei Schritten: Zunächst wird untersucht, ob die einzelnen Variablen tatsächlich Risikofaktoren darstellen, welche die zeitliche Streuung von Aktienrenditen abbilden. Danach wird überprüft, inwieweit diese Faktoren Aktienportfolio-Renditen im Querschnitt erklären können.

Bei den linearen Zeitreihen-Regressionen im CAPM und in verschiedenen Multifaktormodellen zeigt sich analog zum US-amerikanischen, kanadischen und britischen Aktienmarkt, dass ein Dreifaktorenmodell, das die drei Risikofaktoren des Aktienmarktes enthält, eine deutlich höhere Erklärungskraft besitzt als das traditionelle CAPM. Dagegen weisen die zwei Risikofaktoren des Anleihenmarktes in einem Fünffaktorenmodell keinen zusätzlichen Erklä- 
rungsgehalt für die Überschussrendite von Aktienportfolios auf. Gegenüber dem US-amerikanischen und kanadischen Aktienmarkt kann das Dreifaktorenmodell allerdings am deutschen Aktienmarkt die zeitliche Streuung von Renditen weniger gut abbilden. Dagegen werden Aktienportfolio-Renditen im Querschnitt durch die durchschnittlichen Prämien der drei Risikofaktoren in der Bundesrepublik Deutschland wesentlich besser erklärt als in den USA. Bemerkenswert ist dabei, dass am deutschen Aktienmarkt (teilweise im Gegensatz zum USamerikanischen und britischen Aktienmarkt) nicht nur das Dreifaktorenmodell, sondern auch die anderen betrachteten Multifaktormodelle und das CAPM einen hohen Erklärungsgehalt für diese Portfolio-Renditen im Querschnitt aufweisen. 


\title{
Multifaktormodelle zur Erklärung deutscher Aktienrenditen: Eine empirische Analyse
}

\author{
Andreas Ziegler, Elke Eberts, Michael Schröder, Anja Schulz und Richard Stehle*
}

Juli 2003

\begin{abstract}
Zusammenfassung
Dieses Papier untersucht, inwieweit Multifaktormodelle nach Fama/French (1993) am deutschen Aktienmarkt die zeitliche Streuung von Renditen abbilden und Portfolio-Renditen im Querschnitt erklären können. Analog zu vergleichbar angelegten Studien am US-amerikanischen, kanadischen und britischen Aktienmarkt ergibt sich aus den linearen ZeitreihenRegressionen, dass ein Dreifaktorenmodell, das neben der Überschussrendite des Aktienmarktes zwei weitere Risikofaktoren des Aktienmarktes enthält (die sich aus dem Marktwert sowie dem Quotienten aus Buch- und Marktwert ableiten), eine wesentlich höhere Erklärungskraft für die Überschussrendite von Aktienportfolios besitzt als das traditionelle Capital Asset Pricing Model. Dagegen weisen zwei Risikofaktoren des Anleihenmarktes (die sich aus der Zinsstruktur und dem Ausfallrisiko ableiten) in einem Fünffaktorenmodell keinen zusätzlichen Erklärungsgehalt auf. Gegenüber den USA und Kanada kann das Dreifaktorenmodell allerdings für die Bundesrepublik Deutschland die zeitliche Streuung von Aktienrenditen nur schlechter abbilden. Dagegen werden Portfolio-Renditen im Querschnitt am deutschen Aktienmarkt wesentlich besser erklärt als am US-amerikanischen Aktienmarkt.
\end{abstract}

Schlüsselwörter: Multifaktormodelle, CAPM, Aktienrenditen

\footnotetext{
Dr. Andreas Ziegler, Dr. Elke Eberts, Dr. Michael Schröder: Zentrum für Europäische Wirtschaftsforschung, Mannheim. Dipl.-Kffr. Anja Schulz, Prof. Richard Stehle Ph.D.: Lehrstuhl für Bank- und Börsenwesen, Humboldt-Universität zu Berlin.

Wir danken Julie Hausladen, Svenja Engler und Jan-Hendrik Hirsch für hilfreiche Kommentare.
} 


\section{Multifaktormodelle zur Erklärung deutscher Aktienrenditen: Eine empirische Analyse}

\section{Einleitung}

Die systematische Erklärung von Aktienrenditen ist seit langem eine der zentralen Herausforderungen der finanzökonomischen Forschung. In dieser Hinsicht ist bis heute das von Sharpe (1964) und Lintner (1965) entwickelte Capital Asset Pricing Model (CAPM) der verbreitetste Ansatz. Entsprechend dem CAPM hängt die erwartete Rendite eines Aktienportfolios oder einer Aktie in einem linearen Ansatz ausschließlich vom risikolosen Zins und von der entsprechenden erwarteten Risikoprämie des Aktienmarktes ab. Zudem impliziert das CAPM, dass der so genannte "Beta-Faktor" ausreicht, um die erwartete Aktienrendite zu erklären. Allerdings hat eine Vielzahl empirischer Studien schon ab Anfang der 1980er Jahre gezeigt, dass auch andere Faktoren einen Beitrag zur Erklärung von Aktienrenditen liefern können. ${ }^{1}$ Da Einflüsse solcher Faktoren nicht durch das CAPM erfasst werden, werden sie in der finanzökonomischen Literatur häufig als Anomalien bezeichnet. ${ }^{2}$ Die Untersuchungen von $F a$ ma/French $(1992,1993)$ haben jedoch in der jüngeren Vergangenheit die Diskussion um ein zum CAPM alternatives Kapitalmarktmodell verstärkt.

In linearen Querschnitts-Regressionen untersuchen Fama/French (1992) am US-amerikanischen Aktienmarkt eine Reihe verschiedener potentieller Faktoren (wie z.B. das KursGewinn-Verhältnis oder den Verschuldungsgrad) hinsichtlich ihres Effektes auf die durchschnittliche Rendite. Dabei kommen sie zu dem Ergebnis, dass der geschätzte "Beta-Faktor" des CAPM keinen signifikanten Einfluss besitzt. Demgegenüber weisen sie einen signifikant positiven Einfluss eines kleinen Marktwertes sowie eines hohen Quotienten aus Buch- und Marktwert auf die durchschnittliche Aktienrendite nach. Fama und French argumentieren, dass die Prämie für Aktien mit kleinen Marktwerten oder hohen Quotienten aus Buch- und Marktwert eine Kompensation für Risiko darstellt, das durch das CAPM nicht abgedeckt wird. ${ }^{3}$ Darauf aufbauend schätzen Fama/French (1993) in linearen Zeitreihen-Regressionen Multifaktormodelle, die neben der Überschussrendite des Aktienmarktes über den risikolosen

\footnotetext{
Vgl. z.B. Banz (1981), Basu (1983), DeBondt/Thaler (1985) oder Rosenberg et al. (1985).

Vgl. z.B. Fama/French (1996).

Vgl. auch Fama/French (1995, 1996, 1998), Davis et al. (2000).
} 
Zins vier weitere Faktoren zur Erklärung der entsprechenden Überschussrendite von Aktienportfolios einbeziehen. Dabei identifizieren sie neben der Überschussrendite des Aktienmarktes insbesondere zwei zentrale Risikofaktoren des Aktienmarktes: Die Differenz der durchschnittlichen Renditen von Aktiengesellschaften mit einem kleinen und einem großen Marktwert sowie die Differenz der durchschnittlichen Renditen von Aktiengesellschaften mit einem hohen und einem geringen Quotienten aus Buch- und Marktwert. Vergleichbar angelegte Studien zeigen, dass das von Fama/French (1993) am US-amerikanischen Aktienmarkt empirisch bestätigte Dreifaktorenmodell die Überschussrendite von Portfolios auch am kanadischen ${ }^{4}$ und britischen ${ }^{5}$ Aktienmarkt besser erklären kann als das CAPM.

Auch am deutschen Aktienmarkt werden mittlerweile der Marktwert und/oder der Quotient aus Buch- und Marktwert (neben anderen in der Literatur diskutierten Variablen) hinsichtlich des Einflusses auf Renditen von Aktienportfolios oder Aktien analysiert. Dazu werden regelmäßig lineare Querschnitts-Regressionen heran gezogen ${ }^{6}$, bei denen alle untersuchten Aktienportfolios oder Aktien gemeinsam in die Schätzungen einbezogen werden, sei es im Rahmen eines zweistufigen Ansatzes entsprechend Fama/MacBeth (1973) oder im Rahmen von multivariaten Pooling-Verfahren. Im Gegensatz dazu sind (nach unserer Kenntnis) am deutschen Aktienmarkt bisher noch keine umfangreichen linearen Zeitreihen-Regressionen der Überschussrenditen von Aktienportfolios auf die von Fama/French (1993) vorgeschlagenen Risikofaktoren und damit keine empirische Überprüfung ihrer Ergebnisse durchgeführt worden.

Dementsprechend wird in diesem Papier untersucht, ob solche Multifaktormodelle auch am deutschen Aktienmarkt eine höhere Erklärungskraft für die Überschussrendite von Aktienportfolios besitzen als das CAPM. Dazu werden Portfolios auf der Grundlage des Marktwertes sowie des Quotienten aus Buch- und Marktwert der Aktiengesellschaften gebildet. Anlehnend an Fama/French (1993) erfolgt die Analyse in zwei Schritten: ${ }^{7}$ Zunächst wird untersucht, ob die einzelnen Variablen tatsächlich Risikofaktoren darstellen, welche die zeitliche Streuung von Aktienrenditen abbilden. Danach wird überprüft, inwieweit diese Faktoren Aktienportfolio-Renditen im Querschnitt erklären können. Dabei werden ebenso wie bei Berkowitz/Qiu (2001) auch zwei zusätzliche Risikofaktoren des Anleihenmarktes analysiert: Die

4 Vgl. Berkowitz/Qiu (2001).

5 Vgl. Hussain et al. (2002).

6 Vgl. z.B. Sattler (1994), Stehle (1997), Bunke et al. (1998), Wallmeier (2000), Stock (2002), Schulz/Stehle (2002).

$7 \mathrm{Zu}$ beachten ist, dass in diesem Papier ausschließlich die Erklärung der Überschussrendite von Aktienportfolios über den risikolosen Zins analysiert wird. Im Gegensatz dazu betrachten Fama/French (1993) zusätzlich auch die Erklärung der Überschussrendite von Anleihenportfolios. 
Überschussrendite eines Portfolios langfristiger Staatsanleihen über den risikolosen Zins sowie die Differenz der Rendite eines Portfolios langfristiger Unternehmensanleihen und eines Portfolios langfristiger Staatsanleihen. Ein weiterer wichtiger Untersuchungsgegenstand dieses Papiers ist, ob diese beiden Risikofaktoren des Anleihenmarktes am deutschen Aktienmarkt im Gegensatz zum US-amerikanischen und kanadischen Aktienmarkt auch zeitliche Streuungen von Renditen abbilden sowie deren durchschnittliche Prämien einen zusätzlichen Erklärungsgehalt für Portfolio-Renditen im Querschnitt liefern können.

Das vorliegende Papier analysiert alle im amtlichen Handel an der Frankfurter Wertpapierbörse zwischen Dezember 1967 und Juni 1995 notierten Industrieunternehmen, für welche die notwendigen Daten hinsichtlich der monatlichen Aktienrenditen, der Marktwerte und der Buchwerte des Eigenkapitals vorliegen. Demnach konzentriert sich dieses Papier im Gegensatz zur Untersuchung von Wallmeier (2000), der Aktien aus dem amtlichen Handel, dem geregelten Markt und dem Freiverkehr einbezieht, auf das (ehemals) höchste Marktsegment, das wegen seiner hohen Liquidität und starken Überwachung durch Analysten als informationseffizient gilt. Somit ist es für dieses Segment von besonderem Interesse, ob Multifaktormodelle die Überschussrendite von Aktienportfolios besser erklären können als das CAPM.

Bei den linearen Zeitreihen-Regressionen im CAPM und in den Multifaktormodellen zeigt sich, dass das erwähnte Dreifaktorenmodell, das die drei Risikofaktoren des Aktienmarktes enthält, deutlich besser die zeitliche Streuung von Aktienrenditen abbilden und Aktienportfolio-Renditen im Querschnitt erklären kann als das traditionelle CAPM. Dagegen weisen die zwei Risikofaktoren des Anleihenmarktes in einem Fünffaktorenmodell keinen zusätzlichen Erklärungsgehalt für die Überschussrendite von Aktienportfolios auf. Gegenüber dem USamerikanischen und kanadischen Aktienmarkt kann das Dreifaktorenmodell allerdings am deutschen Aktienmarkt die zeitliche Streuung von Renditen weniger gut abbilden. Dagegen werden Portfolio-Renditen im Querschnitt durch die durchschnittlichen Prämien der drei Risikofaktoren wesentlich besser erklärt als am US-amerikanischen Aktienmarkt. Bemerkenswert ist, dass am deutschen Aktienmarkt (teilweise im Gegensatz zum US-amerikanischen und britischen Aktienmarkt) nicht nur das Dreifaktorenmodell, sondern auch die anderen betrachteten Multifaktormodelle und das CAPM in dieser Hinsicht einen hohen Erklärungsgehalt aufweisen. 
Das vorliegende Papier ist wie folgt strukturiert: Im zweiten Abschnitt werden Möglichkeiten der empirischen Überprüfung des CAPM und der verschiedenen Multifaktormodelle erläutert. Im dritten Abschnitt wird die Konstruktion der betrachteten Aktienportfolios dargestellt. Der vierte Abschnitt beschreibt Spezifika der für die Schätzung des CAPM und der Multifaktormodelle verwendeten Daten. Im fünften Abschnitt werden die empirischen Ergebnisse diskutiert. Der sechste Abschnitt fasst schließlich die wichtigsten Ergebnisse zusammen und zieht einige Schlussfolgerungen.

\section{CAPM und Multifaktormodelle}

Zur empirischen Überprüfung des CAPM in linearen Zeitreihen-Regressionen wird die Überschussrendite eines Aktienportfolios (oder auch einer Aktie) über den einperiodigen risikolosen Zins durch die entsprechende Überschussrendite des Aktienmarktes erklärt. Somit gilt ( $i=$ $1, \ldots, I ; t=1, \ldots, T)$ :

$$
r_{i t}-r_{t}^{r f}=\alpha_{i}+\beta_{i 1}\left(r_{m t}-r_{t}^{r f}\right)+\varepsilon_{i t}
$$

Dabei bezeichnen in diesem Papier $r_{i t}$ die monatliche Rendite von Aktienportfolio $i$ und $r_{m t}$ die monatliche Rendite des Aktienmarktes jeweils zum Ende von Monat $t$ sowie $r^{r f}{ }_{t}$ den risikolosen Einmonats-Zins zum Anfang von Monat $t{ }^{8}$ Während $\varepsilon_{i t}$ die stochastische Komponente mit den üblichen Standardannahmen darstellt, bezeichnen $\alpha_{i}$ und $\beta_{i 1}$ die zu schätzenden Parameter.

Bei Multifaktormodellen entsprechend Fama/French (1993) wird in linearen ZeitreihenRegressionen zur Erklärung der Überschussrendite $r_{i t}-r^{r f}{ }_{t}$ mehr als ein Risikofaktor einbezogen. Das in diesem Papier grundlegende Modell ist folgendes Fünffaktorenmodell $(i=1, \ldots, I$; $t=1, \ldots, T)$ :

$$
r_{i t}-r_{t}^{r f}=\alpha_{i}+\beta_{i 1}\left(r_{m t}-r_{t}^{r f}\right)+\beta_{i 2} S M B_{t}+\beta_{i 3} H M L_{t}+\beta_{i 4} T E R M_{t}+\beta_{i 5} D E F_{t}+\varepsilon_{i t}
$$

Dabei stellt $\varepsilon_{i t}$ wiederum die stochastische Komponente dar und $\alpha_{i}, \beta_{i 1}, \beta_{i 2}, \beta_{i 3}, \beta_{i 4}$ sowie $\beta_{i 5}$ bezeichnen die zu schätzenden Parameter. Zur Konstruktion von $S M B_{t}$ (“Small Minus Big”)

\footnotetext{
${ }^{8}$ Bei allen in diesem Papier betrachteten Renditen handelt es sich um diskrete Renditen.
} 
und $H M L_{t}$ ("High Minus Low") werden zunächst Ende Juni eines jeden Jahres für alle betrachteten Aktiengesellschaften der Median des Marktwertes sowie unabhängig davon die 30\%- und 70\%-Perzentile des Quotienten aus Buch- und Marktwert Ende Dezember des voran gehenden Jahres berechnet. ${ }^{9}$ Diese drei Werte bilden die Grundlage für die Anordnung von sechs Aktienportfolios:

S-H: "Small-High", also kleiner Marktwert, hoher Quotient aus Buch- und Marktwert $S$-M: "Small-Medium", also kleiner Marktwert, mittlerer Quotient aus Buch- und Marktwert $S$-L: "Small-Low", also kleiner Marktwert, geringer Quotient aus Buch- und Marktwert $B-H$ : "Big-High", also großer Marktwert, hoher Quotient aus Buch- und Marktwert B-M: "Big-Medium", also großer Marktwert, mittlerer Quotient aus Buch- und Marktwert $B-L$ : "Big-Low", also großer Marktwert, geringer Quotient aus Buch- und Marktwert

Die einzelnen Unternehmen werden dann entsprechend ihrem Marktwert Ende Juni des Jahres sowie entsprechend ihrem Quotienten aus Buch- und Marktwert Ende Dezember des voran gehenden Jahres einem dieser sechs Portfolios zugeordnet und verbleiben dort von Juli des betrachteten Jahres bis Juni des nächsten Jahres. In die Analyse werden somit ausschließlich solche Aktiengesellschaften einbezogen, für die Ende Dezember des voran gehenden Jahres der Markt- und Buchwert sowie Ende Juni des betrachteten Jahres der Marktwert vorliegen. Unbetrachtet bleiben dabei allerdings jene Aktiengesellschaften, die Ende Dezember des voran gehenden Jahres einen negativen Buchwert besitzen. Im Juni des nächsten Jahres werden nach einer erneuten Sortierung auf der Grundlage des Marktwertes sowie des Quotienten aus Buch- und Marktwert sechs neue Portfolios gebildet. Über den gesamten Beobachtungszeitraum werden dann zum Ende von Monat $t$ für alle Aktienportfolios $S-H, S-M, S-L, B-H, B-M$ und $B-L$ die entsprechenden monatlichen Renditen $r^{S-H}, r_{t}^{S-M}, r_{t}^{S-L}, r^{B-H}, r^{B-M}$ und $r^{B-L}$ bestimmt. Diese Berechnung erfolgt mit dem marktwertgewichteten Durchschnitt der monatlichen Aktienrenditen über alle Unternehmen in den jeweiligen Portfolios. Schließlich ermitteln sich die Risikofaktoren des Aktienmarktes folgenderweise:

$$
\begin{aligned}
& S M B_{t}=\left(r^{S-H}{ }_{t}+r^{S-M}{ }_{t}+r^{S-L}{ }_{t}\right) / 3-\left(r^{B-H}{ }_{t}+r^{B-M}{ }_{t}+r^{B-L}{ }_{t}\right) / 3 \\
& H M L_{t}=\left(r^{S-H}{ }_{t}+r^{B-H}{ }_{t}\right) / 2-\left(r^{S-L}{ }_{t}+r^{B-L} t\right) / 2
\end{aligned}
$$

Durch diese Konstruktion der Faktoren soll erreicht werden, dass $S M B_{t}$ weitgehend unbeein-

\footnotetext{
9 Vgl. Fama/French (1993).
} 
flusst vom Quotienten aus Buch- und Marktwert sowie $H M L_{t}$ weitgehend unbeeinflusst vom Marktwert sind.

TERM $M_{t}$ bezeichnet die monatliche Wertänderung $r_{t}^{S}$ eines Portfolios langfristiger Staatsanleihen über den risikolosen Einmonats-Zins $r^{r f}$ und bildet somit die Überschussrendite eines Portfolios von Staatsanleihen ohne Ausfallrisiko ab. $D E F_{t}$ bezeichnet die Differenz der monatlichen Rendite $r_{t}^{U U}$ eines Portfolios langfristiger Unternehmensanleihen und der monatlichen Rendite $r^{U S}{ }_{t}$ eines Portfolios langfristiger Staatsanleihen (jeweils zum Ende von Monat $t$ ). Der Risikofaktor bildet demnach die zeitveränderliche Prämie von Unternehmensanleihen ab. In diesem Papier wird $D E F_{t}$ im Gegensatz zu TERM $M_{t}$ analog zu Berkowitz/Qiu (2001) und entgegen Fama/French (1993) als Differenz zweier Umlaufrenditen definiert ${ }^{10}$, da für die Bundesrepublik Deutschland (BRD) ein Index für Unternehmensanleihen nicht in geeigneter Qualität zur Verfügung steht. Somit gilt für die Risikofaktoren des Anleihenmarktes:

$$
\begin{aligned}
& \operatorname{TERM}_{t}=r_{t}{ }_{t}-r^{r f}{ }_{t} \\
& D E F_{t}=r^{U U}{ }_{t}-r^{U S}{ }_{t}
\end{aligned}
$$

Neben dem CAPM werden im Folgenden ausgehend vom Fünffaktorenmodell weitere Multifaktormodelle analysiert. So werden Zweifaktorenmodelle betrachtet, die ausschließlich die beiden Risikofaktoren $S M B_{t}$ und $H M L_{t}$ des Aktienmarktes sowie ausschließlich die beiden Risikofaktoren $T E R M_{t}$ und $D E F_{t}$ des Anleihenmarktes zur Erklärung der Überschussrendite $r_{i t}-r^{r f}{ }_{t}$ der Aktienportfolios einbeziehen. Insbesondere wird auch das in den Studien von $F a$ ma/French (1993), Berkowitz/Qiu (2001) und Hussain et al. (2002) gegenüber dem CAPM sich als vorteilhaft erweisende Dreifaktorenmodell geschätzt, das neben der Überschussrendite $r_{m t}-r^{r f}$ des Aktienmarktes zusätzlich $S M B_{t}$ und $H M L_{t}$ als erklärende Variablen enthält. Fama und French argumentieren, dass $S M B_{t}$ und $H M L_{t}$ jeweils ein systematisches und damit nicht diversifizierbares Risiko des gesamten Aktienmarktes abbilden. ${ }^{11}$ Die beiden Risikofaktoren haben für die USA einen Bezug zum Unternehmensgewinn. Aktiengesellschaften mit einem kleinen Marktwert oder einem hohen Quotienten aus Buch- und Marktwert weisen typischerweise eine relativ geringe Profitabilität auf. Daran anknüpfend weisen die beiden $\mathrm{Au}-$ toren darauf hin, dass die von ihnen formulierten und empirisch bestätigten Multifaktormo-

\footnotetext{
${ }^{10}$ Vgl. dazu Abschnitt 4.

11 Vgl. auch Fama/French (1995, 1996, 1998), Davis et al. (2000)
} 
delle mit Gleichgewichtsmodellen wie zum Beispiel dem intertemporalen CAPM von Merton (1973) vereinbar sind.

In der jüngeren Vergangenheit ist eine lebhafte Diskussion um die korrekte Interpretation der Ergebnisse von Fama und French im Gange. In Wallmeier (2000) findet sich eine relativ umfassende und aktuelle Darstellung der bisherigen Resultate dieser Diskussion. Dabei hat sich die Vermutung, dass die Ergebnisse von Fama/French (1993) Zufallsprodukte eines intensiven so genannten "data snooping"-Prozesses darstellen, nicht als richtig erwiesen, da das Dreifaktorenmodell auch für unterschiedliche Beobachtungszeiträume und für verschiedene Staaten empirisch bestätigt wird. Nicht zuletzt tragen auch die Resultate dieses Papiers mit der Untersuchung am deutschen Aktienmarkt zur weiteren Untermauerung des Dreifaktorenmodells bei. Dies schließt allerdings nicht aus, dass sich in Zukunft ein anderes Kapitalmarktmodell als dasjenige von Fama und French als sinnvoller erweisen könnte. Daniel/Titman (1997) zeigen zum Beispiel, dass ein auf Unternehmenscharakteristika basierendes Modell dem auf Risikofaktoren aufbauenden Modell nach Fama/French (1993) am US-amerikanischen Aktienmarkt überlegen sein könnte. Dies hätte erhebliche Auswirkungen auf das Verständnis von Kapitalmärkten, da die Ergebnisse von Daniel und Titman eher auf Verhaltensanomalien der Investoren hinweisen, die nicht mit Modellen des intertemporalen Marktgleichgewichts vereinbar sind. ${ }^{12}$ Allerdings scheinen bislang die Multifaktormodelle von $\mathrm{Fa}$ ma und French empirisch gut bestätigt zu sein.

\section{Konstruktion der Aktienportfolios}

Im Hinblick auf die Untersuchung der CAPM-Anomalien bezüglich des Marktwertes sowie des Quotienten aus Buch- und Marktwert erfolgt auch in diesem Papier anlehnend an $\mathrm{Fa}$ ma/French (1993), Berkowitz/Qiu (2001) und Hussain et al. (2002) die Konstruktion der Aktienportfolios, deren Überschussrendite erklärt werden soll, auf der Grundlage des Marktwertes sowie des Quotienten aus Buch- und Marktwert. Diese Sortierung der Portfolios ist besonders im Hinblick auf die Untersuchung der Effekte des Marktwertes sowie des Quotienten aus Buch- und Marktwert geeignet, da die Streuung der beiden Variablen zwischen den gebildeten Aktienportfolios nicht wesentlich reduziert wird. Analog zu Berkowitz/Qiu (2001) werden insgesamt $I=16$ Aktienportfolios analysiert. Aufgrund der deutlich geringeren Anzahl bör-

\footnotetext{
${ }^{12}$ Vgl. zur aktuellen Diskussion auch Davis et al. (2000) und Daniel et al. (2001).
} 
sennotierter deutscher Aktiengesellschaften (insbesondere gegenüber US-amerikanischen) werden damit nicht wie in Fama/French (1993) oder Hussain et al. (2002) 25 Aktienportfolios betrachtet. Mit dem Beobachtungszeitraum von Juli 1968 bis Juni 1995 werden insgesamt $T=324$ Monate in die Analyse einbezogen. Im Gegensatz dazu betrachten Fama/French (1993), Berkowitz/Qiu (2001) und Hussain et al. (2002) die Zeiträume von Juli 1963 bis Dezember 1991 (342 Monate), von Februar 1982 bis Juni 1999 (209 Monate) und von Februar 1975 bis Juni 1999 (293 Monate).

Die Konstruktion der 16 Aktienportfolios erfolgt analog zu Berkowitz/Qiu (2001) systematisch in ähnlicher Weise wie die Konstruktion von $S M B_{t}$ und $H M L_{t}$. Für alle betrachteten Aktiengesellschaften werden Ende Juni eines jeden Jahres des Beobachtungszeitraumes die Quartile des Marktwertes sowie unabhängig davon die Quartile des Quotienten aus Buch- und Marktwert Ende Dezember des voran gehenden Jahres berechnet. Die einzelnen Unternehmen werden dann entsprechend ihrem Marktwert Ende Juni des Jahres sowie entsprechend ihrem Quotienten aus Buch- und Marktwert Ende Dezember des voran gehenden Jahres einem dieser aus den sechs Quartilen gebildeten 16 Portfolios zugeordnet und verbleiben dort von Juli des betrachteten Jahres bis Juni des nächsten Jahres. Betrachtet werden erneut lediglich Unternehmen, für die Ende Dezember des voran gehenden Jahres der Markt- und Buchwert sowie Ende Juni des betrachteten Jahres der Marktwert vorliegt. Unbetrachtet bleiben dagegen wieder jene Aktiengesellschaften mit einem negativen Buchwert des Eigenkapitals. Im Juni des nächsten Jahres werden nach einer erneuten Anordnung auf der Grundlage des Marktwertes sowie des Quotienten aus Buch- und Marktwert wieder 16 neue Aktienportfolios gebildet.

$\mathrm{Zu}$ beachten ist, dass ein Unternehmen aufgrund dieser Konstruktion zwischen Juli des betrachteten Jahres und Juni des nächsten Jahres zeitweise oder dauerhaft aus dem Aktienportfolio heraus fällt, falls für ein oder mehrere Monate keine Daten zur Berechnung der Aktienrendite zur Verfügung stehen, zum Beispiel weil die Aktien des Unternehmens nicht mehr notiert werden. Die Berechnung der monatliche Rendite $r_{i t}$ von Aktienportfolio $i=1, \ldots, 16$ erfolgt mit dem Durchschnitt der monatlichen Aktienrendite über alle zum jeweiligen Zeitpunkt in den Portfolios enthaltenen Aktiengesellschaften (jeweils berechnet am Ende jeden Monats $t=1, \ldots, 324)$. Analog zu Fama/French (1993) und Berkowitz/Qiu (2001) wird dabei der marktwertgewichtete Durchschnitt der monatlichen Aktienrendite verwendet. Im Gegensatz dazu betrachten Hussain et al. (2002) den gleichgewichteten Durchschnitt. Im weiteren 
Verlauf des Papiers werden die 16 Portfolios entsprechend ihrem Marktwert sowie ihrem Quotienten aus Buch- und Marktwert mit 1-1 ("Small-Low") ${ }^{13}, \ldots, 1-4$ ("Small-High") $)^{14}, \ldots$,

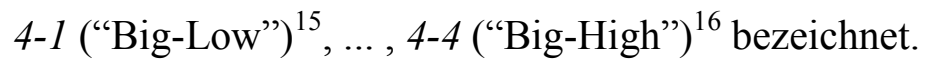

Bei dieser unabhängigen Portfoliobildung enthalten einige der 16 Aktienportfolios wegen der geringen Anzahl börsennotierter deutscher Aktiengesellschaften in einzelnen Monaten des Beobachtungszeitraumes nur eine sehr kleine Anzahl an Unternehmen (die geringste Anzahl beträgt Drei). Um daraus potentielle für die Schätzungen folgende Verzerrungen zu überprüfen, ist deshalb auch eine abhängige Portfoliobildung in 16 Aktienportfolios auf der Grundlage des Marktwertes sowie des Quotienten aus Buch- und Marktwert betrachtet worden. Dabei werden (Ende Juni eines jeden Jahres) zunächst die Quartile des Marktwertes berechnet und die Unternehmen dann entsprechend ihrem Marktwert in die resultierenden vier Portfolios eingeteilt. Danach werden für die Aktiengesellschaften jedes einzelnen dieser vier Portfolios getrennt voneinander die Quartile des Quotienten aus Buch- und Marktwert (Ende Dezember des voran gehenden Jahres) generiert und die Aktiengesellschaften entsprechend ihrem Quotienten aus Buch- und Marktwert den jeweils vier resultierenden Subportfolios zugeordnet. Mit dieser Vorgehensweise umfassen die 16 Aktienportfolios über den gesamten Beobachtungszeitraum eine weitgehend identische Anzahl an Unternehmen.

Darüber hinaus ist auch eine unabhängige Portfoliobildung in lediglich neun statt 16 Aktienportfolios auf der Grundlage des Marktwertes sowie des Quotienten aus Buch- und Marktwert durchgeführt worden. Dadurch wird erreicht, dass die Anzahl der Unternehmen in den einzelnen Portfolios über den Beobachtungszeitraum nicht so gering wird (die geringste Anzahl beträgt hier Acht) wie bei der Betrachtung von 16 Aktienportfolios. Allerdings haben sich bei der Schätzung des CAPM und der verschiedenen Multifaktormodelle auf der Grundlage dieser drei unterschiedlichen Portfoliobildungen keine systematisch unterschiedlichen Resultate ergeben. Deshalb wird im Folgenden lediglich die unabhängige Portfoliobildung in 16 Aktienportfolios (auf der Grundlage des Marktwertes sowie des Quotienten aus Buch- und Marktwert) betrachtet, so dass eine bessere Vergleichbarkeit der Schätzergebnisse mit den Studien von Fama/French (1993), Berkowitz/Qiu (2001) und Hussain et al. (2002) gewährleistet ist.

\footnotetext{
13 D.h. kleiner Marktwert, geringer Quotient aus Buch- und Marktwert.

14 D.h. kleiner Marktwert, hoher Quotient aus Buch- und Marktwert.

15 D.h. großer Marktwert, geringer Quotient aus Buch- und Marktwert.

16 D.h. großer Marktwert, hoher Quotient aus Buch- und Marktwert.
} 


\section{Daten}

In diesem Papier werden für die Bildung der 16 Aktienportfolios sowie der Variablen $S M B_{t}$ und $H_{M}$ alle im amtlichen Handel an der Frankfurter Wertpapierbörse zwischen Dezember 1967 und Juni 1995 notierten Industrieunternehmen betrachtet, für welche die notwendigen Daten hinsichtlich der monatlichen Aktienrenditen, der Marktwerte und der Buchwerte vorliegen. Banken und Versicherungen bleiben unberücksichtigt, da diese sich bei der Bewertung durch den Markt von Industrieunternehmen unterscheiden und speziellen Rechnungslegungsvorschriften unterliegen. Die Ermittlung des Buchwertes des Eigenkapitals erfolgt auf der Grundlage der Deutschen Finanzdatenbank (DFDB), die unter anderem die Einzelabschlüsse börsennotierter Aktiengesellschaften enthält. ${ }^{17}$ Eine Berechnung des bilanziellen Eigenkapitals auf der Basis von Konzernabschlüssen wird nicht vorgenommen, da für den Beobachtungszeitraum keine miteinander vergleichbaren Konzernabschlüsse vorliegen. Die Umsetzung der für Konzernabschlüsse geltenden 7. EG-Richtlinie durch das BilanzrichtlinienGesetz (BiRiLiG) von 1985 bewirkte überwiegend materielle Änderungen der Bilanzposition. Im Gegensatz dazu hatte die für Einzelabschlüsse geltende 4. EG-Richtlinie vorrangig Umgliederungsvorgänge ohne bedeutende materielle Auswirkungen zur Konsequenz.

Der berechnete Buchwert des Eigenkapitals wird Ende Dezember eines jeden Jahres des Beobachtungszeitraums zum entsprechenden Marktwert der Aktiengesellschaft in Beziehung gesetzt. Dabei wird unterstellt, dass die Bewertung des Kapitalmarktes auch auf den Teil des Grundkapitals zutrifft, der nicht an der Börse zugelassen ist. Der Marktwert des Eigenkapitals entspricht dem Produkt von Aktienkurs und Aktienanzahl Ende Juni eines jeden Jahres des Beobachtungszeitraumes. Falls ein Unternehmen mehrere Aktiengattungen an der Börse notiert hat, berechnet sich der Marktwert des Eigenkapitals aus der Summe der Marktwerte der einzelnen Aktiengattungen. Der gesamte Marktwert des Unternehmens wird in den Zeitreihen-Regressionen ausschließlich den monatlichen Renditen der Stammaktie gegenüber gestellt. Diese monatlichen Aktienrenditen sowie die zur Berechnung der Marktwerte benötigten Daten entstammen dabei einer mehrfach überprüften Datenbank des Lehrstuhls für Bank- und Börsenwesen der Humboldt-Universität zu Berlin. Die Aktienrenditen werden aus der Perspektive eines deutschen Anlegers mit einem Einkommensteuersatz von 0\% durch Einbeziehung von Kursveränderungen, Bruttodividenden und sonstiger geldwerter Vorteile bestimmt. $^{18}$

\footnotetext{
17 Für Einzelheiten vgl. Schulz/Stehle (2002).

18 Vgl. dazu ausführlich Stehle/Hartmond (1991).
} 
Für die Berechnung der monatlichen Rendite $r_{m t}$ des Aktienmarktes werden gegenüber der Bildung der Aktienportfolios alle zwischen (Ende) Juni 1968 und (Ende) Juni 1995 im amtlichen Handel an der Frankfurter Wertpapierbörse notierten Unternehmen betrachtet. Damit sind sowohl Banken und Versicherungen bei der Bestimmung dieser Renditen berücksichtigt als auch jene Aktiengesellschaften, die Ende Dezember eines Jahres einen negativen Buchwert des Eigenkapitals aufweisen. Als risikoloser Einmonats-Zins $r^{r f}{ }_{t}$ zwischen (Anfang) Juli 1968 und (Anfang) Juni 1995 wird der Monats-Durchschnitt des Einmonats-Geldes am Frankfurter Bankplatz verwendet. Die monatliche Rendite $r_{t}^{S}$ eines Portfolios langfristiger Staats-

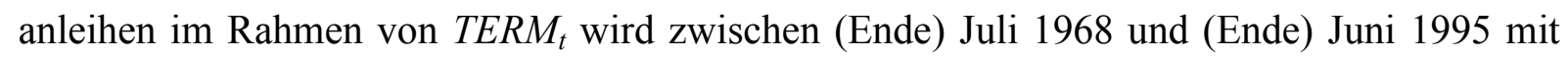
Hilfe des REX-Performanceindex (REXP) für Bundesanleihen mit synthetischen Restlaufzeiten von einem bis zehn Jahren berechnet. Dagegen wird $r_{t}^{U S}$ im Rahmen von $D E F_{t}$ für diesen Zeitraum mit der Umlaufrendite von Bundesanleihen abgebildet. ${ }^{19}$ Schließlich entstammt die monatliche Rendite $r_{t}^{U U}$ eines Portfolios langfristiger Unternehmensanleihen zwischen (Ende) Juli 1968 und (Ende) Juni 1995 der Berechnung der Umlaufrendite inländischer Industrieobligationen durch die Deutsche Bundesbank.

\section{Ergebnisse}

\subsection{Deskriptive Statistiken}

Im oberen Teil von Tabelle 1 sind zusammenfassende Statistiken der betrachteten Risikofaktoren $r_{m t}-r_{t}^{r f}, S M B_{t}, H M L_{t}, T E R M_{t}$ und $D E F_{t}$ am deutschen Aktienmarkt für den Zeitraum zwischen Juli 1968 und Juni 1995 abgebildet. Dabei werden jeweils die arithmetischen Mittel und Standardabweichungen sowie Pearsonsche Korrelationskoeffizienten ausgewiesen. Daneben enthält der obere Teil der Tabelle die arithmetischen Mittel und Standardabweichungen der einzelnen Komponenten $r_{m t}, r^{r f}{ }_{t}, r_{t}^{S}, r^{U S}{ }_{t}$ und $r^{U U}{ }_{t}$ der erklärenden Variablen in den verschiedenen Regressionsmodellen. ${ }^{20}$ Die durchschnittliche monatliche Rendite des deutschen Aktienmarktes zwischen Juli 1968 und Juni 1995 beträgt $0.801 \%$ und die entsprechende durchschnittliche monatliche Überschussrendite beträgt $0.265 \%$. Demgegenüber lauten die korrespondierenden Werte am US-amerikanischen Aktienmarkt ${ }^{21}$ von Juli 1963 bis Dezember

\footnotetext{
${ }^{19}$ Vgl. auch Abschnitt 2.

20 Im Folgenden wird ein arithmetisches Mittel oder ein Pearsonscher Korrelationskoeffizient als nicht signifikant von Null verschieden bezeichnet, falls die Nullhypothese im entsprechenden Parametertest auf den Wert Null zu einem Signifikanzniveau von $10 \%$ nicht verworfen werden kann.

21 Vgl. dazu im Folgenden Fama/French (1993).
} 
1991 0.97\% und 0.43\% sowie am kanadischen Aktienmarkt ${ }^{22}$ von Februar 1982 bis Juni 1999 $1.051 \%$ und $0.384 \%$. Lediglich für die USA ist aber das arithmetische Mittel der monatlichen Überschussrendite zu einem Signifikanzniveau von 10\% von Null verschieden.

Am deutschen Aktienmarkt ist für $H M L_{t}$ das arithmetische Mittel (und damit die durchschnittliche Prämie für diesen Risikofaktor) in Höhe von $0.402 \%$ zu einem Signifikanzniveau von $1 \%$ von Null verschieden. Dieser Wert korrespondiert mit ähnlichen hohen Werten von $0.40 \%$ und $0.426 \%$ am US-amerikanischen und kanadischen Aktienmarkt. Allerdings ist für Kanada das arithmetische Mittel nicht signifikant von Null verschieden. Demnach deutet sich für die BRD bei dieser Variablen nach der Kontrolle von Differenzen im Marktwert eine deutlich positive Relation zwischen dem Quotienten aus Buch- und Marktwert sowie der Aktienrendite an. Dagegen scheint bei der Analyse von $S M B_{t}$ am deutschen Aktienmarkt mit einem arithmetischen Mittel in Höhe von $0.083 \%$ nach der Kontrolle von Differenzen im Quotienten aus Buch- und Marktwert keine starke Relation zwischen dem Marktwert und der Aktienrendite vorzuliegen, zumal dieser Durchschnitt nicht signifikant von Null verschieden ist. Dies unterscheidet sich von den entsprechenden arithmetischen Mitteln in Höhe von $0.27 \%$ und $0.427 \%$ am US-amerikanischen und kanadischen Aktienmarkt, die jeweils zu einem Signifikanzniveau von $10 \%$ von Null verschieden sind.

Für $D E F_{t}$ ist das arithmetische Mittel am deutschen Aktienmarkt trotz eines geringen Wertes von $0.032 \%$ zu einem Signifikanzniveau von weit unter $1 \%$ von Null verschieden. Am USamerikanischen und kanadischen Aktienmarkt lauten die entsprechenden Werte $0.02 \%$ und $0.063 \%$. Aber nur für Kanada kann die Nullhypothese, dass das arithmetische Mittel Null ist, auch zu einem Signifikanzniveau von weit unter 1\% verworfen werden. Die jeweils extrem geringen Standardabweichungen bei $D E F_{t}$ für die BRD und Kanada im Vergleich zu den USA dürften damit zusammen hängen, dass für die Berechnung dieser Variablen in diesem Papier und bei Berkowitz/Qiu (2001) entgegen dem Vorgehen von Fama/French (1993) Umlaufrenditen von Portfolios langfristiger Unternehmens- und Staatsanleihen verwendet werden. Für TERM $M_{t}$ ist das arithmetische Mittel am deutschen Aktienmarkt in Höhe von $0.057 \%$ ebenso wie am US-amerikanischen Aktienmarkt in Höhe von $0.06 \%$ nicht signifikant von Null verschieden. Demgegenüber lautet das arithmetische Mittel für Kanada 0.528\% und ist dort zu einem Signifikanzniveau von $1 \%$ von Null verschieden.

\footnotetext{
${ }^{22}$ Vgl. dazu im Folgenden Berkowitz/Qiu (2001).
} 
Die Pearsonschen Korrelationskoeffizienten zwischen $r_{m t}-r^{r f}$ und TERMt, zwischen $S M B_{t}$ und $T E R M_{t}$ sowie zwischen $T E R M_{t}$ und $D E F_{t}$ sind für die BRD mit Werten von 0.315, -0.209 sowie $0.224 \mathrm{zu}$ einem Signifikanzniveau von $1 \%$, sowie zwischen $r_{m t}-r^{r f}$ und $H M L_{t}$ mit einem Wert von 0.102 zu einem Signifikanzniveau von 10\% von Null verschieden. Dabei ergibt sich eine relativ starke positive Korrelation zwischen $r_{m t}-r^{r f}{ }_{t}$ und TERM in ähnlicher Weise auch für die USA und Kanada (die entsprechenden Werte lauten dort 0.34 und 0.327). Bei den anderen Korrelationen zeigen sich dagegen teilweise starke Differenzen zwischen diesen drei Aktienmärkten. Besonders erwähnenswert ist der Korrelationskoeffizient zwischen $r_{m t}-r^{r f}{ }_{t}$ und $S M B_{t}$, der am US-amerikanischen Aktienmarkt den Wert 0.32 und am kanadischen den Wert 0.008 besitzt. Am deutschen Aktienmarkt liegt dagegen ein negativer Wert in Höhe von -0.597 vor, der auch zu einem Signifikanzniveau von 1\% von Null verschieden ist. Schließlich ergeben sich für die BRD ebenso wie für die USA, Kanada und Großbritannien ${ }^{23}$ (dort für den Zeitraum von Februar 1975 bis Juni 1999) hinsichtlich des Korrelationskoeffizienten zwischen $S M B_{t}$ und $H M L_{t}$ konstruktionsbedingt ${ }^{24}$ geringe betragsmäßige Werte in Höhe von $-0.001,-0.08,-0.008$ und 0.08 .

Im unteren Teil von Tabelle 1 sind die arithmetischen Mittel und Standardabweichungen der abhängigen Variablen in den Regressionsmodellen, das heißt der Überschussrenditen $r_{i t}-r^{r f}{ }_{t}$ der $i=1, \ldots, 16$ Aktienportfolios, abgebildet. Dabei zeigt sich hinsichtlich der durchschnittlichen monatlichen Überschussrenditen am deutschen Aktienmarkt zwischen Juli 1968 und Juni 1995 insgesamt bei allen 16 Aktienportfolios eine Bandbreite zwischen 0.002\% (Portfolio 4-1) und $0.668 \%$ (Portfolio 2-4). Im Vergleich dazu ist das Spektrum am US-amerikanischen (zwischen 0.32\% und 1.05\% von Juli 1963 bis Dezember 1991 bei 25 Portfolios), kanadischen (zwischen $0.24 \%$ und 1.55\% von Februar 1982 bis Juni 1999 bei 16 Portfolios) sowie britischen Aktienmarkt (zwischen 0.41\% und 1.65\% von Februar 1975 bis Juni 1999 bei 25 Portfolios) höher. Wegen teilweise sehr niedriger Werte sind am deutschen Aktienmarkt auch nur die arithmetischen Mittel der monatlichen Überschussrenditen bei Portfolio 2-4 zu einem Signifikanzniveau von 1\%, bei Portfolio 4-4 zu einem Signifikanzniveau von 5\% sowie bei Portfolio 1-4 und Portfolio 3-4 zu einem Signifikanzniveau von 10\% von Null verschieden.

Mit zunehmendem Quotienten aus Buch- und Marktwert in den Aktienportfolios steigen für

\footnotetext{
${ }^{23}$ Vgl. dazu im Folgenden Hussain et al. (2002).

24 Vgl. Abschnitt 2.
} 
die BRD ebenso wie für die USA und Großbritannien tendenziell die durchschnittlichen monatlichen Überschussrenditen an. Analog zu den Ergebnissen für $H M L_{t}$ liegt damit (auch nach der Kontrolle von Differenzen im Marktwert) eine positive Relation zwischen dem Quotienten aus Buch- und Marktwert sowie der Aktienrendite vor. Demgegenüber ist am deutschen Aktienmarkt analog zu den Ergebnissen für $S M B_{t}$ auch bei den durchschnittlichen monatlichen Überschussrenditen von Portfolios (auch nach der Kontrolle von Differenzen im Quotienten aus Buch- und Marktwert) keine systematische Relation zum Marktwert festzustellen. Mit zunehmendem Marktwert in den Aktienportfolios ergeben sich sowohl wachsende als auch sinkende arithmetische Mittel. Dagegen zeigt sich für die USA, Kanada und Großbritannien eine negative Relation zwischen dem Marktwert und der durchschnittlichen monatlichen Überschussrendite von Aktienportfolios. 
Tabelle 1: Deskriptive Statistiken (in Prozent pro Monat) der erklärenden und abhängigen Variablen in den verschiedenen linearen Regeressionsmodellen: Deutscher Aktienmarkt von Juli 1968 bis Juni 1995 ( $t=1, \ldots, 324$ Monate)

\begin{tabular}{|c|c|c|c|c|c|c|c|}
\hline \multicolumn{8}{|c|}{ Erklärende Variablen } \\
\hline \multirow{2}{*}{ Variable } & \multirow{2}{*}{$\begin{array}{l}\text { Arith- } \\
\text { metisches } \\
\text { Mittel }\end{array}$} & \multirow{2}{*}{$\begin{array}{c}\text { Standard- } \\
\text { ab- } \\
\text { weichung }\end{array}$} & \multicolumn{5}{|c|}{ Pearsonscher Korrelationskoeffizient } \\
\hline & & & $r_{m t}-r_{t}^{r f}$ & $S M B_{t}$ & $H M L_{t}$ & $T E R M_{t}$ & $D E F_{t}$ \\
\hline$r_{m t}$ & $0.801^{* * *}$ & 4.697 & & & & & \\
\hline$r^{r f}{ }_{t}$ & $0.536^{* * *}$ & 0.200 & & & & & \\
\hline$r_{t}^{S}$ & $0.593^{* * *}$ & 1.146 & & & & & \\
\hline$r^{U S}{ }_{t}$ & $0.616^{* * *}$ & 0.105 & & & & & \\
\hline$r_{t}^{U U}$ & $0.648^{* * *}$ & 0.103 & & & & & \\
\hline$r_{m t}-r_{t}^{r f}$ & 0.265 & 4.717 & 1 & & & & \\
\hline$S M B_{t}$ & 0.083 & 2.578 & $-0.597^{* * *}$ & 1 & & & \\
\hline$H M L_{t}$ & $0.402^{* * *}$ & 2.302 & $0.102^{*}$ & -0.001 & 1 & & \\
\hline$T E R M_{t}$ & 0.057 & 1.151 & $0.315^{* * *}$ & $-0.209^{* * *}$ & 0.030 & 1 & \\
\hline$D E F_{t}$ & $0.032^{* * *}$ & 0.022 & 0.036 & -0.066 & -0.011 & $0.224^{* * *}$ & 1 \\
\hline \multicolumn{8}{|c|}{$\begin{array}{l}\text { Abhängige Variablen, d.h. Überschussrenditen } r_{i t}-r_{t}^{r f} \text { der } i=1, \ldots, 16 \text { Aktienportfolios: } \\
\text { Arithmetisches Mittel (Standardabweichung) }\end{array}$} \\
\hline \multirow{2}{*}{$\begin{array}{l}\text { Markt- } \\
\text { werte }\end{array}$} & \multicolumn{7}{|c|}{ Quotienten aus Buch- und Marktwert } \\
\hline & \multicolumn{2}{|c|}{1 (Low) } & 2 & \multicolumn{2}{|r|}{3} & \multicolumn{2}{|c|}{4 (High) } \\
\hline 1 (Small) & 0.224 & (4.721) & $(3.88$ & 0.329 & $(4.464)$ & $0.589^{*}$ & (5.459) \\
\hline 2 & 0.215 & (3.915) & $(4.38$ & 0.317 & $(4.495)$ & $0.668^{* * *}$ & $(4.602)$ \\
\hline 3 & 0.157 & $(4.070)$ & $(4.41$ & 0.236 & (4.699) & $0.574^{*}$ & (5.565) \\
\hline 4 (Big) & 0.002 & $(5.710)$ & $(5.05$ & 0.287 & $(5.083)$ & $0.595^{* *}$ & (5.257) \\
\hline
\end{tabular}

Anmerkungen:

*, ** bzw. ${ }^{* *}$ bedeutet, dass die Nullhypothese eines arithmetischen Mittels oder eines Pearsonschen Korrelationskoeffizienten von Null zu einem Signifikanzniveau von 10\%, 5\% bzw. $1 \%$ verworfen werden kann.

\subsection{Lineare Zeitreihen-Regressionen}

In Tabelle 2 bis Tabelle 6 sind die Ergebnisse der Schätzungen des CAPM und der betrachteten Multifaktormodelle am deutschen Aktienmarkt abgebildet. Anlehnend an Fama/French (1993) erfolgt die Analyse der Resultate in zwei Schritten. Zunächst wird in Abschnitt 5.2.1 bis 5.2.4 untersucht, ob die Variablen $r_{m t}-r^{r f}{ }_{t}, S M B_{t}, H M L_{t}, T E R M_{t}$ und $D E F_{t}$ für die BRD tatsächlich Risikofaktoren darstellen, welche die zeitliche Streuung von Aktienrenditen abbilden. Die Überprüfung dieser Fragestellung in Tabelle 2 bis Tabelle 5 erfolgt durch die Betrachtung der geschätzten Parameter der einzelnen Risikofaktoren und der korrigierten $R^{2}$. Als 
insignifikant wird dabei im Folgenden der Einfluss einer erklärenden Variablen beschrieben, falls der Betrag des entsprechenden z-Wertes kleiner als 1.65 ist. $^{25}$ In einem zweiten Schritt wird in Abschnitt 5.2.5 untersucht, inwieweit die durchschnittlichen Prämien der fünf Risikofaktoren am deutschen Aktienmarkt Portfolio-Renditen im Querschnitt erklären können. Die Überprüfung dieser Fragestellung in Tabelle 6 erfolgt mit der Analyse der geschätzten Konstanten $\alpha_{i}$.

\subsubsection{CAPM}

In Tabelle 2 sind die geschätzten Parameter der erklärenden Variablen und die korrigierten $R^{2}$ des CAPM am deutschen Aktienmarkt abgebildet. Analog zu den Ergebnissen am US-amerikanischen (für 25 Portfolios), kanadischen (für 16 Portfolios) und britischen Aktienmarkt (für 25 Portfolios), zeigen sich insgesamt hohe z-Werte für den Parameter $\beta_{i l}$, so dass die Überschussrendite $r_{m t}-r^{r f}{ }_{t}$ des Aktienmarktes bei allen untersuchten $i=1, \ldots, 16$ Portfolios zu einem Signifikanzniveau von deutlich unter 1\% einen Einfluss auf die Überschussrendite $r_{i t}-r^{r f}{ }_{t}$ besitzt. Wie für Großbritannien, Kanada und die USA nehmen dabei die z-Werte mit wachsendem Marktwert in den Aktienportfolios stark zu. Darüber hinaus ist (erneut analog zum britischen, aber im Gegensatz zum US-amerikanischen Aktienmarkt) mit zunehmendem Marktwert (und gleich bleibendem Quotienten aus Buch- und Marktwert) in den Portfolios ein Anstieg der entsprechenden Schätzwerte zu verzeichnen. Zunehmende Schätzwerte für $\beta_{i 1}$ ergeben sich am deutschen Aktienmarkt zudem tendenziell (außer bei den Portfolios mit den größten Marktwerten), wenn (bei gleich bleibendem Marktwert) der Quotient aus Buch- und Marktwert steigt. Bemerkenswert sind (ähnlich wie für Großbritannien mit Werten zwischen 0.48 und 1.10) die insgesamt relativ geringen Schätzwerte zwischen 0.470 (bei Portfolio 1-2) und 1.074 (bei Portfolio 4-1). Dagegen ergeben sich für Kanada sowie für die USA Werte zwischen 0.787 und 1.097 sowie zwischen 0.84 und 1.42, wobei sich am US-amerikanischen Aktienmarkt tendenziell mit kleinerem Marktwert sowie geringerem Quotienten aus Buchund Marktwert in den Portfolios höhere Schätzwerte zeigen.

Die korrigierten $R^{2}$ variieren am deutschen Aktienmarkt bei allen Portfolios zwischen 0.229 (bei Portfolio 1-1) und 0.844 (bei Portfolio 4-2). Dabei steigen diese $R^{2}$ (wie für Kanada mit

\footnotetext{
${ }^{25}$ D.h. der entsprechende Parameter ist in diesem Fall lediglich zu einem Signifikanzniveau von über 10\% von Null verschieden.
} 
Werten zwischen 0.106 und 0.867 , für Großbritannien mit Werten zwischen 0.21 und 0.92 sowie in abgeschwächter Form für die USA mit Werten zwischen 0.61 und 0.92) mit zunehmendem Marktwert in den Aktienportfolios deutlich an. Dagegen zeigen sich für die BRD im Gegensatz zu den USA, für die sich mit sinkendem Quotienten aus Buch- und Marktwert (und gleich bleibendem Marktwert) in den Portfolios eine schwache Zunahme dieser Werte ergibt, keine systematischen Einflüsse von Seiten dieses Quotienten. Bei der Betrachtung der Schätzund z-Werte der Parameter der erklärenden Variablen sowie der korrigierten $R^{2}$ scheint das CAPM somit an allen vier untersuchten Aktienmärkten die zeitliche Streuung von Renditen gut abzubilden. Ebenso wie am kanadischen, britischen und US-amerikanischen Aktienmarkt verbleiben, gemessen am $R^{2}$, aber auch am deutschen Aktienmarkt insbesondere für kleine Marktwerte in den Portfolios zeitliche Variationen von Renditen, die durch andere Risikofaktoren abgebildet werden könnten.

Tabelle 2: Lineare Zeitreihen-Regressionen der Überschussrenditen $r_{i t}-r^{r f}{ }_{t}$ der $i=1, \ldots, 16$ Aktienportfolios auf die Überschussrendite $r_{m t}-r^{r f}{ }_{t}$ des Aktienmarktes: Deutscher Aktienmarkt von Juli 1968 bis Juni 1995 ( $t=1, \ldots, 324$ Monate)

\begin{tabular}{|c|c|c|c|c|}
\hline \multicolumn{5}{|c|}{$r_{i t}-r_{t}^{r f}=\alpha_{i}+\beta_{i l}\left(r_{m t}-r_{t}^{r f}\right)+\varepsilon_{i t}$} \\
\hline \multirow{3}{*}{$\begin{array}{l}\text { Markt- } \\
\text { werte }\end{array}$} & \multicolumn{4}{|c|}{ Quotienten aus Buch- und Marktwert } \\
\hline & 1 (Low) & 2 & 3 & 4 (High) \\
\hline & \multicolumn{4}{|c|}{ Schätzwert (z-Wert) für $\beta_{i 1}$} \\
\hline 1 (Small) & $0.482^{* * *} \quad(9.85)$ & $0.470^{* * *}(12.47)$ & $0.617^{* * *}(15.43)$ & $0.742^{* * *}(14.98)$ \\
\hline 2 & $0.615^{* * *}(19.77)$ & $0.659^{* * *}(18.06)$ & $0.685^{* * *}(18.55)$ & $0.710^{* * *}(19.03)$ \\
\hline 3 & $0.674^{* * *}(22.47)$ & $0.727^{* * *}(22.06)$ & $0.766^{* * *}(21.56)$ & $0.900^{* * *}(21.20)$ \\
\hline \multirow[t]{2}{*}{4 (Big) } & $1.074^{* * *}(34.54)$ & $0.985^{* * *}(41.82)$ & $0.989^{* * *}(41.37)$ & $0.999^{* * *}(36.30)$ \\
\hline & \multicolumn{4}{|c|}{ Korrigiertes $R^{2}$} \\
\hline 1 (Small) & 0.229 & 0.324 & 0.423 & 0.409 \\
\hline 2 & 0.547 & 0.502 & 0.515 & 0.528 \\
\hline 3 & 0.609 & 0.601 & 0.590 & 0.581 \\
\hline 4 (Big) & 0.787 & 0.844 & 0.841 & 0.803 \\
\hline
\end{tabular}

Anmerkungen:

${ }^{*},{ }^{*}$ bzw. ${ }^{* * *}$ bedeutet, dass $r_{m t}-r^{r f}{ }_{t}$ nach dem zweiseitigen Parametertest auf den Wert Null zu einem Signifikanzniveau von $10 \%, 5 \%$ bzw. $1 \%$ einen Einfluss auf $r_{i t}-r^{r f}{ }_{t}$ besitzt. 


\subsubsection{Zweifaktorenmodelle}

In Tabelle 3 sind die geschätzten Parameter der erklärenden Variablen und die korrigierten $R^{2}$ der Zweifaktorenmodelle am deutschen Aktienmarkt abgebildet. Der obere Teil der Tabelle enthält die Resultate der Zeitreihen-Regressionen der Überschussrenditen $r_{i t}-r^{r f}{ }_{t} \operatorname{der} i=$ 1,...,16 Aktienportfolios auf die beiden Risikofaktoren $S M B_{t}$ und $H M L_{t}$ des Aktienmarktes. Dabei zeigt sich analog zum US-amerikanischen und kanadischen Aktienmarkt ${ }^{26}$ tendenziell mit sinkendem Marktwert in den Portfolios eine teilweise deutliche Zunahme der Schätzwerte für $\beta_{i 2}$ sowie mit wachsendem Quotienten aus Buch- und Marktwert eine teilweise deutliche Zunahme der Schätzwerte für $\beta_{i 3}$. Allerdings liegen die Schätzwerte für $\beta_{i 2}$ klar unterhalb, die Schätzwerte für $\beta_{i 3}$ aber (fast durchweg) klar oberhalb derjenigen für Kanada. Gegenüber den USA zeigt sich dieser Unterschied in noch stärkerem Ausmaß. Insbesondere ergeben sich am deutschen Aktienmarkt (außer bei Portfolio 1-1) negative Schätzwerte für $\beta_{i 2}$. Bei den Aktienportfolios mit den beiden größten Marktwerten ist dieser Parameter zu einem Signifikanzniveau von weit unter $1 \%$ von Null verschieden. Dagegen sind die Schätzwerte für $\beta_{i 3}$ (außer bei Portfolio 2-1 und 4-1) positiv. Dabei ist dieser Parameter tendenziell vor allem bei Aktienportfolios mit hohen Quotienten aus Buch- und Marktwert signifikant von Null verschieden.

Das korrigierte $R^{2}$ variiert am deutschen Aktienmarkt bei allen 16 Portfolios zwischen -0.001 und 0.440. Dabei nehmen die Werte ähnlich wie bei der Schätzung des CAPM mit steigendem Marktwert (und gleich bleibendem Quotienten aus Buch- und Marktwert) in den Aktienportfolios zu. Daneben zeigt sich auch mit wachsendem Quotienten aus Buch- und Marktwert (und gleich bleibendem Marktwert) in den Portfolios eine Tendenz zu höheren $R^{2}$. Im Gegensatz dazu sinken die entsprechenden Werte für die USA mit zunehmendem Marktwert (und gleich bleibendem Quotienten aus Buch- und Marktwert) sowie tendenziell mit steigendem Quotienten aus Buch- und Marktwert (und gleich bleibendem Marktwert) in den dort untersuchten 25 Aktienportfolios. Vor allem aber ist für die USA insgesamt das Niveau der Werte bei Variationen zwischen 0.04 und 0.65 höher. Für Kanada variieren die Werte zwischen -0.001 und 0.483 etwa auf dem Niveau der BRD, wobei sich tendenziell mit kleinen Marktwerten in den dort untersuchten 16 Aktienportfolios höhere $R^{2}$ ergeben. Insgesamt scheinen $S M B_{t}$ und $H M L_{t}$ in einem Zweifaktorenmodell am deutschen Aktienmarkt einige zeitliche

\footnotetext{
${ }^{26} \mathrm{Zu}$ beachten ist, dass in der Studie von Hussain et al. (2002) für den britischen Aktienmarkt keine Zweifakto-
} renmodelle betrachtet werden. 
Streuungen von Renditen abbilden zu können, wenn auch in geringerem Umfang als am USamerikanischen Aktienmarkt. Im Gegensatz zu den USA und Kanada können diese beiden Risikofaktoren des Aktienmarktes für die BRD bei der Betrachtung der Schätz- und z-Werte der Parameter der erklärenden Variablen sowie der korrigierten $R^{2}$ tendenziell lediglich zur Abbildung der zeitlichen Streuung in den Aktienportfolios mit großen Marktwerten sowie hohen Quotienten aus Buch- und Marktwert beitragen.

Im unteren Teil von Tabelle 3 sind die Ergebnisse der Zeitreihen-Regressionen der Überschussrenditen $r_{i t}-r^{r f}{ }_{t}$ der $i=1, \ldots, 16$ Aktienportfolios auf die beiden Risikofaktoren TERM und $D E F_{t}$ des Anleihenmarktes abgebildet. Analog zum kanadischen Aktienmarkt zeigt sich auch am deutschen Aktienmarkt mit steigendem Marktwert in den Portfolios tendenziell eine Zunahme der Schätzwerte für $\beta_{i 4}$. Dabei besitzen die Schätzwerte für die BRD, die zwischen 0.342 (bei Portfolio 1-2) und 1.277 (bei Portfolio 4-4) liegen, insgesamt ein höheres Niveau als für Kanada. Für die USA zeigen sich dagegen vergleichsweise geringere Streuungen bei den einzelnen Aktienportfolios. Bemerkenswert ist aber, dass TERM $A_{t}$ am deutschen Aktienmarkt bei allen Portfolios zu einem Signifikanzniveau von 10\% sowie außer bei Portfolio 1-1, 1-2 und 1-4 zu einem Signifikanzniveau von $1 \%$ einen Einfluss auf $r_{i t}-r^{r f}{ }_{t}$ besitzt. Insofern kann gefolgert werden, dass der Risikofaktor TERM ${ }_{t}$ des Anleihenmarktes am deutschen Aktienmarkt analog zum kanadischen und US-amerikanischen Aktienmarkt (bei letzterem ist der entsprechende Parameter bei allen Aktienportfolios zu einem Signifikanzniveau von deutlich unter $1 \%$ von Null verschieden) zumindest teilweise die zeitliche Streuung von Renditen abbilden kann.

Diese Schlussfolgerung lässt sich für die BRD für $D E F_{t}$ nicht ziehen. Bei allen Aktienportfolios ergeben sich bei betragsmäßig sehr hohen Schätzwerten für $\beta_{i 5}$ äußerst hohe geschätzte Standardabweichungen der geschätzten Parameter mit der Konsequenz, dass $\beta_{i 5}$ lediglich bei Portfolio 1-4 zu einem Signifikanzniveau von 5\% und bei Portfolio 1-1 zu einem Signifikanzniveau von $10 \%$ von Null verschieden ist. Diese unplausiblen Schätzergebnisse dürften vor allem mit der Berechnung von $D E F_{t}$ durch Umlaufrenditen von Portfolios langfristiger Unternehmens- und Staatsanleihen zusammen hängen. Zu bemerken ist, dass am kanadischen Aktienmarkt bei ähnlicher Konstruktion von $D E F_{t}$ teilweise geringere geschätzte Standardabweichungen der entsprechend geschätzten Parameter vorliegen, so dass immerhin bei fünf der dort untersuchten 16 Aktienportfolios zu einem Signifikanzniveau von 5\% ein Einfluss auf $r_{i t}-r^{r f}{ }_{t}$ nachgewiesen werden kann. Zu betonen ist aber, dass $\beta_{i 5}$ am US-amerikanischen Ak- 
tienmarkt bei abweichender Konstruktion von $D E F_{t}$ bei allen dort betrachteten 25 Portfolios zu einem Signifikanzniveau von deutlich unter 1\% von Null verschieden ist. Die entsprechenden Schätzwerte nehmen dabei mit sinkendem Marktwert in den Aktienportfolios zu. Festzuhalten ist damit, dass der in diesem Papier konstruierte Risikofaktor $D E F_{t}$ des Anleihenmarktes am deutschen Aktienmarkt offensichtlich keine zeitliche Streuung von Renditen abbilden kann.

Verstärkt wird diese Argumentation durch die Resultate in Bezug auf die korrigierten $R^{2}$. Trotz des häufig signifikanten Einflusses von TERM $M_{t}$ auf $r_{i t}-r^{r f}{ }_{t}$ ergeben sich am deutschen Aktienmarkt bei allen Portfolios sehr kleine Werte zwischen 0.004 (bei Portfolio 1-2) und 0.070 (bei Portfolio 4-3 und 4-4). Zwar sind die entsprechenden $R^{2}$ am kanadischen sowie US-amerikanischen Aktienmarkt mit Werten zwischen -0.009 und 0.176 sowie zwischen 0.06 und 0.21 auch auf einem niedrigen Niveau, aber insbesondere für die USA durchschnittlich deutlich höher als für die BRD. Dabei steigen die korrigierten $R^{2}$ an allen drei Aktienmärkten und insbesondere am kanadischen mit wachsendem Marktwert (und gleich bleibendem Quotienten aus Buch- und Marktwert) in den Portfolios tendenziell an. Insgesamt scheinen somit $T E R M_{t}$ und $D E F_{t}$ in einem Zweifaktorenmodell lediglich partiell die zeitliche Streuung von Aktienrenditen abbilden zu können. $\mathrm{Zu}$ bemerken ist dabei, dass Zeitreihen-Regressionen ${ }^{27}$ der Überschussrenditen $r_{i t}-r_{t}^{r f}$ alleine auf TERM $M_{t}$ oder alleine auf $D E F_{t}$ trotz der (entsprechend Tabelle 1) signifikant positiven Korrelation dieser beiden Risikofaktoren nur wenig systematisch unterschiedliche Schätzergebnisse liefern wie Zeitreihen-Regressionen auf $T E R M_{t}$ und $D E F_{t}$ zusammen. Festzuhalten ist damit, dass $S M B_{t}$ und $H M L_{t}$ in einem Zweifaktorenmodell für die BRD wie für die USA und Kanada bei der Betrachtung der Schätz- und zWerte der Parameter der erklärenden Variablen sowie der korrigierten $R^{2}$ offensichtlich besser die zeitliche Streuung von Aktienrenditen abbilden können als $T E R M_{t}$ und $D E F_{t}$ in einem Zweifaktorenmodell.

\footnotetext{
${ }^{27}$ Die entsprechenden Schätzergebnisse sind in diesem Papier nicht gesondert ausgewiesen.
} 
Tabelle 3: Lineare Zeitreihen-Regressionen der Überschussrenditen $r_{i t}-r^{r f}{ }_{t}$ der $i=1, \ldots, 16$ Aktienportfolios auf $S M B_{t}$ und $H M L_{t}$ sowie auf TERM $M_{t}$ und DEF $F_{t}$ Deutscher Aktienmarkt von Juli 1968 bis Juni 1995 ( $t=1, \ldots, 324$ Monate)



Anmerkungen:

*, ** bzw. *** bedeutet, dass nach dem zweiseitigen Parametertest auf den Wert Null die jeweilige erklärende Variable zu einem Signifikanzniveau von $10 \%, 5 \%$ bzw. $1 \%$ einen Einfluss auf $r_{i t}-r^{r f}{ }_{t}$ besitzt. 


\subsubsection{Dreifaktorenmodell}

In Tabelle 4 sind die geschätzten Parameter der erklärenden Variablen und die korrigierten $R^{2}$ des Dreifaktorenmodells am deutschen Aktienmarkt abgebildet, das heißt die Tabelle enthält die Resultate der Zeitreihen-Regressionen der Überschussrenditen $r_{i t}-r^{r f}{ }_{t} \operatorname{der} i=1, \ldots, 16 \mathrm{Ak}-$ tienportfolios auf die drei Risikofaktoren $r_{m t}-r^{r f}{ }_{t}, S M B_{t}$ und $H M L_{t}$ des Aktienmarktes. Analog zur Schätzung des Zweifaktorenmodells, bei dem lediglich $S M B_{t}$ und $H M L_{t}$ einbezogen werden, ergeben sich mit sinkendem Marktwert in den Portfolios tendenziell eine Zunahme der Schätzwerte für $\beta_{i 2}$ sowie mit wachsendem Quotienten aus Buch- und Marktwert ein deutlicher Anstieg der Schätzwerte für $\beta_{i 3}$. Demgegenüber schwächt sich der Effekt einer Zunahme der Schätzwerte für $\beta_{i 1}$ vor allem mit wachsendem Marktwert (bei gleich bleibendem Quotienten aus Buch- und Marktwert) und teilweise auch mit wachsendem Quotienten aus Buchund Marktwert (bei gleich bleibendem Marktwert) in den Aktienportfolios im Vergleich zur Schätzung des CAPM ab.

Hinzuweisen ist auf die Verschiebungen in den Niveaus der Schätzwerte für $\beta_{i 1}, \beta_{i 2}$ und $\beta_{i 3}$ gegenüber der Schätzung des CAPM und des Zweifaktorenmodells. Für $\beta_{i 2}$ zeigt sich bei allen Aktienportfolios eine deutliche Zunahme der Schätzwerte. Daraus folgt, dass die Schätzwerte für $\beta_{i 2}$ außer bei den Portfolios mit dem größten Marktwert positiv sind. Darüber hinaus steigen auch die Schätzwerte für $\beta_{i 1}$ bei Variationen zwischen 0.689 (bei Portfolio 1-2) und 1.067 (bei Portfolio 4-1) außer bei den Portfolios mit dem größten Marktwert teilweise deutlich an. Dieses Resultat dürfte maßgeblich durch die (entsprechend Tabelle 1) starke negative Korrelation zwischen $r_{m t}-r^{r f}$ und $S M B_{t}$ beeinflusst sein, die offensichtlich dazu führt, dass die entsprechenden Schätzwerte der Parameter im CAPM und im Zweifaktorenmodell nach unten verzerrt sind, eine typische Auswirkung des Auslassens relevanter erklärender Variablen. Im Gegensatz dazu ergibt sich für $\beta_{i 3}$ bei allen Aktienportfolios ein Rückgang der Schätzwerte gegenüber der Schätzung des Zweifaktorenmodells. Allerdings ist das Ausmaß dieser Veränderung im Vergleich zur Veränderung für $\beta_{i 2}$ geringer. Dieses Resultat korrespondiert (entsprechend Tabelle1) mit einer nur schwachen positiven Korrelation zwischen $r_{m t}-r_{t}^{r f}$ und $H M L_{t}$.

Ebenso wie bei der Schätzung des CAPM besitzt die Überschussrendite $r_{m t}-r^{r f}$ des Aktienmarktes auch bei der Schätzung des Dreifaktorenmodells bei allen 16 Aktienportfolios zu einem Signifikanzniveau von weit unter $1 \%$ einen Einfluss auf $r_{i t}-r^{r f}$. Gegenüber der Schät- 
zung des Zweifaktorenmodells ergibt sich am deutschen Aktienmarkt bei einer höheren Anzahl von 14 Aktienportfolios, dass $\beta_{i 2}$ zu einem Signifikanzniveau von $1 \%$ von Null verschieden ist. Lediglich bei Portfolio 4-1 und Portfolio 4-2 kann kein signifikanter Einfluss von $S M B_{t}$ auf $r_{i t}-r^{r f}{ }_{t}$ nachgewiesen werden. In Bezug auf $H M L_{t}$ zeigt sich gegenüber der Schätzung des Zweifaktorenmodells, dass der Parameter $\beta_{i 3}$ für eine gleich hohe Anzahl von elf Aktienportfolios zu einem Signifikanzniveau von 10\% von Null verschieden ist.

Bei der Betrachtung der Schätz- und z-Werte der Parameter der erklärenden Variablen lässt sich also am deutschen Aktienmarkt folgern, dass der partiell starke Einfluss der Risikofaktoren $S M B_{t}$ und $H M L_{t}$ auf $r_{i t}-r^{r f}$ bei der Schätzung des Zweifaktorenmodells im Rahmen der Schätzung des Dreifaktorenmodells nicht durch den Einfluss von $r_{m t}-r^{r f}{ }_{t}$ überdeckt wird. Damit kann das Dreifaktorenmodell und somit die zusätzliche Einbeziehung von $S M B_{t}$ und $H M L_{t}$ besser die zeitliche Streuung von Aktienrenditen abbilden als das CAPM. Bemerkenswert ist in diesem Zusammenhang insbesondere die Bedeutung von $S M B_{t}$, da sich aus der deskriptiven Analyse entsprechend Tabelle 1 kein Zusammenhang zwischen dem Marktwert und der Aktienrendite heraus kristallisiert hat. Weiter verstärkt wird die Vorteilhaftigkeit des Dreifaktorenmodells gegenüber dem CAPM bei der Betrachtung der korrigierten $R^{2}$. Im Vergleich zu den Schätzergebnissen im CAPM (in Tabelle 2, aber auch im Zweifaktorenmodell im oberen Teil von Tabelle 3) zeigen sich bei allen 16 Aktienportfolios höhere $R^{2}$, insbesondere bei eher kleineren Marktwerten in den Portfolios. Die Werte variieren dabei zwischen 0.422 (bei Portfolio 1-1) und 0.865 (bei Portfolio 4-4). Tendenziell sind mit zunehmendem Marktwert in den Aktienportfolios höhere $R^{2}$ zu erkennen.

Die Vorteilhaftigkeit des Dreifaktorenmodells gegenüber dem CAPM am deutschen Aktienmarkt steht im Einklang mit den Studien am US-amerikanischen, kanadischen und britischen Aktienmarkt. An diesen Aktienmärkten ergeben sich bei allen jeweils untersuchten 25 oder 16 Portfolios bei der Schätzung des Dreifaktorenmodells meist deutlich höhere korrigierte $R^{2}$ als bei der Schätzung des CAPM oder des Zweifaktorenmodells, bei dem lediglich $S M B_{t}$ und $H M L_{t}$ einbezogen werden. Dabei bewegen sich für Kanada die entsprechenden Werte zwischen 0.250 und 0.876 bei vielen Aktienportfolios auf einem ähnlichen Niveau wie für die BRD. Im Gegensatz dazu variieren die $R^{2}$ für Großbritannien zwischen 0.49 und 0.94 sowie für die USA zwischen 0.83 und 0.97. Vor allem am US-amerikanischen Aktienmarkt liegen damit diese Werte (insbesondere bei Portfolios mit kleinem Marktwert) deutlich oberhalb derjenigen am deutschen Aktienmarkt. Bei der alleinigen Betrachtung der korrigierten $R^{2}$ 
kann demnach das Dreifaktorenmodell vor allem für die USA, zum Teil aber auch für Großbritannien offensichtlich noch besser die zeitliche Streuung von Aktienrenditen abbilden als für die BRD oder Kanada.

Die Schätzwerte für $\beta_{i 1}$ zwischen 0.91 und 1.18 am US-amerikanischen Aktienmarkt sowie zwischen 0.80 und 1.17 am britischen Aktienmarkt variieren bei allen untersuchten 25 Portfolios deutlich enger um den Wert Eins als bei der Schätzung des CAPM und damit auch etwas enger als bei der Schätzung des Dreifaktorenmodells am deutschen Aktienmarkt. Am kanadischen Aktienmarkt streuen die entsprechenden Werte zwischen 0.760 und 1.099. Insbesondere aber besitzt die Überschussrendite $r_{m t}-r^{r f}$ des Aktienmarktes auch an diesen drei Aktienmärkten durchweg zu einem Signifikanzniveau von weit unter $1 \%$ einen Einfluss auf $r_{i t}-r^{r f}$. Darüber hinaus bewirkt ein sinkender Marktwert in den Aktienportfolios eine Zunahme der Schätzwerte für $\beta_{i 2}$ sowie ein zunehmender Quotient aus Buch- und Marktwert eine Zunahme der Schätzwerte für $\beta_{i 3}$. Dabei ergeben sich am britischen Aktienmarkt bei allen Portfolios positive Schätzwerte für $\beta_{i 2}$, während diese Werte am US-amerikanischen und kanadischen ebenso wie am deutschen Aktienmarkt bei Portfolios mit dem größten Marktwert kleiner als Null sind. Dagegen sind die Schätzwerte für $\beta_{i 3}$ bei Portfolios mit dem geringsten Quotienten aus Buch- und Marktwert (außer bei einem Portfolio am britischen Aktienmarkt) an allen vier Aktienmärkten negativ. Allerdings ergeben sich für die BRD, USA und Kanada vereinzelt auch bei Aktienportfolios mit höherem Quotienten aus Buch- und Marktwert Werte kleiner als Null.

Bemerkenswert sind auch die z-Werte für $\beta_{i 2}$ und $\beta_{i 3}$. Für die USA, Großbritannien und Kanada ist $\beta_{i 2}$ außer bei jeweils einem Aktienportfolio zu einem Signifikanzniveau von meist deutlich unter $1 \%$ von Null verschieden. Insbesondere am US-amerikanischen und britischen Aktienmarkt ergeben sich dabei maximale z-Werte von 52.85 und 32.08. Der Parameter $\beta_{i 3}$ ist am britischen und insbesondere US-amerikanischen Aktienmarkt häufiger zu einem Signifikanzniveau von $1 \%$ von Null verschieden als am deutschen oder kanadischen Aktienmarkt. Lediglich bei Schätzwerten für $\beta_{i 3}$ nahe Null kann an den beiden erst genannten Aktienmärkten teilweise kein signifikanter Einfluss von $H M L_{t}$ auf $r_{i t}-r^{r f}{ }_{t}$ nachgewiesen werden. Insofern deuten nicht nur die korrigierten $R^{2}$, sondern auch die Schätz- und z-Werte der Parameter der erklärenden Variablen darauf hin, dass dieses Dreifaktorenmodell am US-amerikanischen und teilweise am britischen Aktienmarkt noch besser die zeitliche Streuung von Renditen abbilden kann als am deutschen oder kanadischen Aktienmarkt. 
Tabelle 4: Lineare Zeitreihen-Regressionen der Überschussrenditen $r_{i t}-r^{r f}{ }_{t}$ der $i=1, \ldots, 16$ Aktienportfolios auf die Überschussrendite $r_{m t}-r^{r f}{ }_{t}$ des Aktienmarktes, $S M B_{t}$ und $H M L_{t}$ : Deutscher Aktienmarkt von Juli 1968 bis Juni 1995 ( $t=1, \ldots, 324$ Monate)

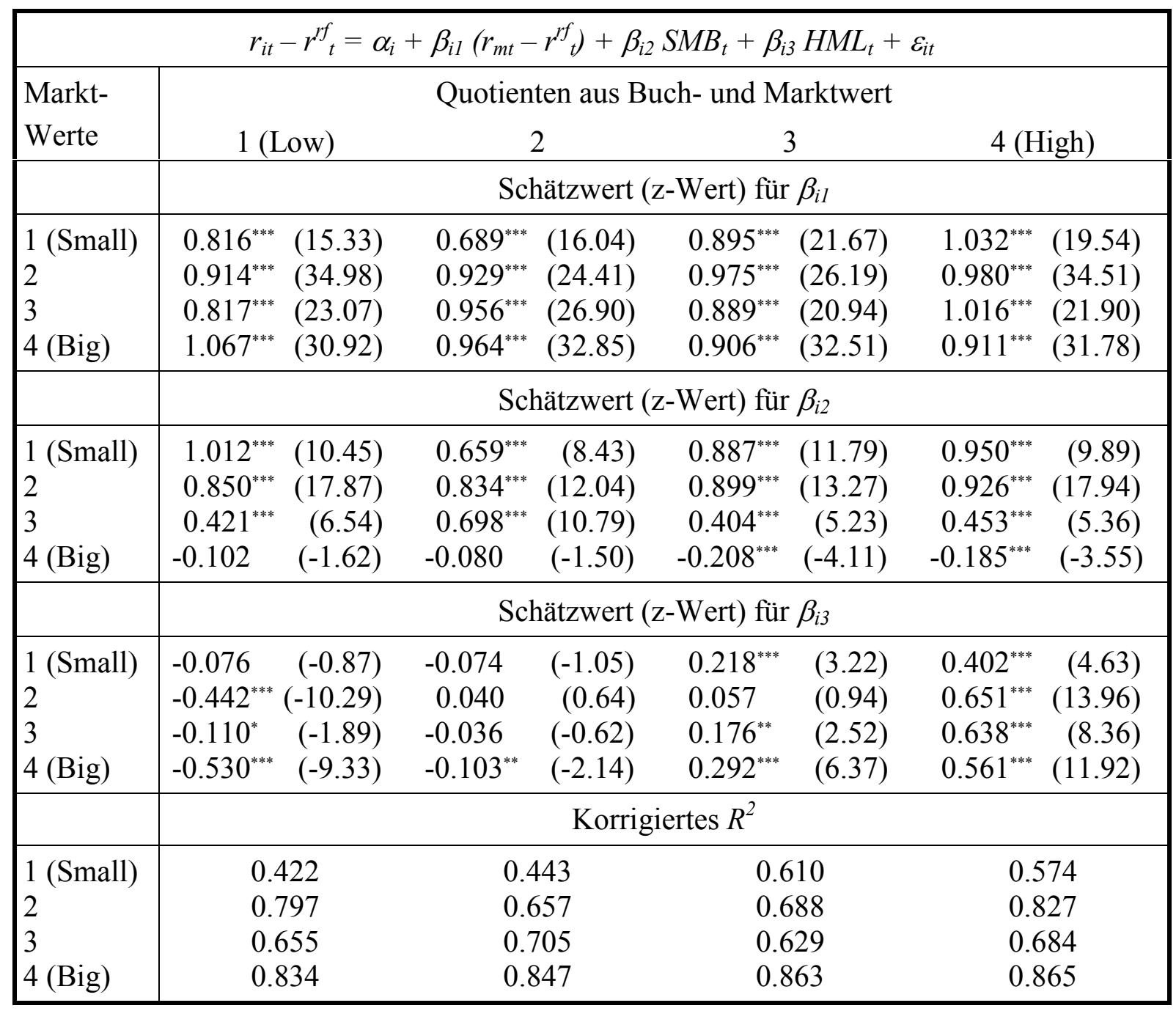

Anmerkungen:

*, ** bzw. *** bedeutet, dass nach dem zweiseitigen Parametertest auf den Wert Null die jeweilige erklärende Variable zu einem Signifikanzniveau von $10 \%, 5 \%$ bzw. $1 \%$ einen Einfluss auf $r_{i t}-r^{r f}{ }_{t}$ besitzt.

\subsubsection{Fünffaktorenmodell}

In Tabelle 5 sind schließlich die geschätzten Parameter der erklärenden Variablen und die korrigierten $R^{2}$ des grundlegenden Fünffaktorenmodells am deutschen Aktienmarkt abgebildet. Die Tabelle enthält somit die Ergebnisse der Zeitreihen-Regressionen der Überschussrenditen $r_{i t}-r_{t}^{r f}$ der $i=1, \ldots, 16$ Aktienportfolios auf alle fünf betrachteten Risikofaktoren $r_{m t}-r_{t}^{r f}, S M B_{t}, H M L_{t}, T E R M_{t}$ und $D E F_{t}$ des Aktien- und Anleihenmarktes. Ein wesentliches Resultat ist die Analogie zu den Resultaten für die Parameter $\beta_{i 1}, \beta_{i 2}$ und $\beta_{i 3}$ bei der Schätzung des Dreifaktorenmodells (entsprechend Tabelle 4). Sowohl die Schätz- als auch die z-Werte 
der Parameter der erklärenden Variablen sind im Fünffaktoren- und Dreifaktorenmodell bei allen Aktienportfolios nahezu identisch (lediglich bei Portfolio 4-1 ist $\beta_{i 2}$ im Fünffaktorenmodell, nicht aber im Dreifaktorenmodell zu einem Signifikanzniveau von 10\% von Null verschieden). Analog zeigen sich zwischen diesen beiden Multifaktormodellen bei den korrigierten $R^{2}$ mit Werten zwischen 0.424 (bei Portfolio 1-1) und 0.864 (bei Portfolio 4-3 und 4-4) im Fünffaktorenmodell bei allen Portfolios nur extrem geringe Unterschiede (und bei Portfolio 2-1 und 2-2 sogar Identitäten). Insofern gelten für die Schätzung des Fünffaktorenmodells sämtliche Aussagen aus Abschnitt 5.2.3 für die Schätzung des Dreifaktorenmodells hinsichtlich des Vergleichs der Schätz- und z-Werte für $\beta_{i 1}, \beta_{i 2}$ und $\beta_{i 3}$ sowie der korrigierten $R^{2}$ mit der Schätzung des CAPM und des Zweifaktorenmodells, bei dem $S M B_{t}$ und $H M L_{t}$ einbezogen werden.

Demgegenüber ergeben sich bei den Schätz- und z-Werten für $\beta_{i 4}$ vehemente Unterschiede zwischen dem Fünffaktorenmodell und dem Zweifaktorenmodell, bei dem die Risikofaktoren $T E R M_{t}$ und $D E F_{t}$ des Anleihenmarktes einbezogen werden (entsprechend dem unteren Teil von Tabelle 3). Im Fünffaktorenmodell sind die Schätzwerte für $\beta_{i 4}$ bei allen 16 Aktienportfolios meist deutlich geringer, so dass diese außer bei Portfolio 3-4 durchweg negativ sind, während sie im Zweifaktorenmodell ausnahmslos größer als Null sind. Vor allem aber ergeben sich vehemente Veränderungen bei den z-Werten. Gegenüber dem meist stark signifikanten Einfluss von TERM $M_{t}$ auf $r_{i t}-r^{r f}{ }_{t}$ bei der Schätzung des Zweifaktorenmodells ist $\beta_{i 4}$ nur noch bei sechs der 16 Aktienportfolios zu einem Signifikanzniveau von 10\% und davon nur noch bei zwei Portfolios zu einem Signifikanzniveau von $1 \%$ von Null verschieden. Für $\beta_{i 5}$ zeigen sich wie im Zweifaktorenmodell bei allen Aktienportfolios äußerst hohe geschätzte Standardabweichungen der geschätzten Parameter bei zumindest partiell betragsmäßig sehr hohen Schätzwerten, so dass $D E F_{t}$ lediglich bei Portfolio 2-3 und Portfolio 1-4 zu einem Signifikanzniveau von 5\% einen Einfluss auf $r_{i t}-r^{r f}$ besitzt. Auch diese recht unplausiblen Schätzergebnisse dürften vor allem durch die Berechnung von $D E F_{t}$ mit Umlaufrenditen von Portfolios langfristiger Unternehmens- und Staatsanleihen begründet sein.

$\mathrm{Zu}$ bemerken ist, dass sich bei den Zeitreihen-Regressionen ${ }^{28}$ der Überschussrenditen $r_{i t}-r^{r f}{ }_{t}$ auf $r_{m t}-r^{r f}{ }_{t}, S M B_{t}, H M L_{t}$ und TERM $M_{t}$ gegenüber der Schätzung des Fünffaktorenmodells bei allen 16 Aktienportfolios nahezu identische Schätz- und z-Werte für $\beta_{i l}, \beta_{i 2}, \beta_{i 3}$ und korri-

\footnotetext{
${ }^{28}$ Die entsprechenden Schätzergebnisse sind in diesem Papier nicht gesondert ausgewiesen.
} 
gierte $R^{2}$ sowie weitgehend analoge Schätz- und z-Werte für $\beta_{i 4}$ ergeben. Demnach können die Risikofaktoren TERM $M_{t}$ und $D E F_{t}$ des Anleihenmarktes bei der Betrachtung der entsprechenden Schätz- und z-Werte sowie der korrigierten $R^{2}$ im Fünffaktorenmodell über die Risikofaktoren des Aktienmarktes hinaus nicht zusätzliche zeitliche Streuungen von Renditen am deutschen Aktienmarkt abbilden. Für $D E F_{t}$ kann dieses Ergebnis schon bei der Schätzung des Zweifaktorenmodells abgeleitet werden. Der meist stark signifikante Einfluss von $T E R M_{t}$ auf $r_{i t}-r^{r f}{ }_{t}$ im Zweifaktorenmodell verschwindet dagegen weitgehend durch die Einbeziehung von $r_{m t}-r_{t}^{r f}, S M B_{t}$ und $H M L_{t}$ im Fünffaktoren- oder auch Vierfaktorenmodell, so dass dieser Risikofaktor des Anleihenmarktes offensichtlich durch die drei Risikofaktoren des Aktienmarktes absorbiert wird. Schon das Dreifaktorenmodell bildet demnach am deutschen Aktienmarkt die zeitliche Streuung von Renditen sehr gut ab.

Diese Ergebnisse stehen ebenfalls weitgehend im Einklang mit den entsprechenden Studien am US-amerikanischen und kanadischen Aktienmarkt. ${ }^{29}$ Auch dort ergeben sich bei den Schätz- und z-Werten für $\beta_{i 1}, \beta_{i 2}$ und $\beta_{i 3}$ sowie bei den korrigierten $R^{2}$ bei allen Portfolios keine substantiellen Unterschiede zwischen dem Fünffaktoren- und dem Dreifaktorenmodell. Bemerkenswert ist dabei, dass die Schätzwerte für $\beta_{i 4}$ und $\beta_{i 5}$ im Fünffaktorenmodell für die USA bei allen Aktienportfolios gegenüber dem Zweifaktorenmodell deutlich geringer sind und damit nahe bei Null liegen. Daraus folgt, dass im Gegensatz zur Schätzung des Zweifaktorenmodells sowohl $\beta_{i 4}$ als auch $\beta_{i 5}$ bei den meisten der 25 betrachteten Aktienportfolios nicht signifikant von Null verschieden sind. Auf der Grundlage weiter gehender Untersuchungen argumentieren zwar Fama/French (1993), dass $T E R M_{t}$ und $D E F_{t}$ dennoch zusätzliche zeitliche Streuungen von Aktienrenditen abbilden können. Aber auch sie kommen zu dem Ergebnis, dass der Einfluss dieser beiden Risikofaktoren des Anleihenmarktes bei der Schätzung des Fünffaktorenmodells von der Überschussrendite $r_{m t}-r_{t}^{r f}$ des Aktienmarktes absorbiert wird. Insofern kann gefolgert werden, dass schon das Dreifaktorenmodell im Vergleich zum Fünffaktorenmodell nicht nur am deutschen und kanadischen, sondern auch am USamerikanischen Aktienmarkt ganz wesentlich die zeitliche Streuung von Renditen abbilden kann.

\footnotetext{
${ }^{29}$ In der Studie von Hussain et al. (2002) für den britischen Aktienmarkt werden keine Fünffaktorenmodelle betrachtet.
} 
Tabelle 5: Lineare Zeitreihen-Regressionen der Überschussrenditen $r_{i t}-r^{r f}$ der $i=1, \ldots, 16$ Aktienportfolios auf die Überschussrendite $r_{m t}-r^{r f}{ }_{t}$ des Aktienmarktes, $S M B_{t}, H M L_{t}, T E R M_{t}$ und DEF $F_{t}$ Deutscher Aktienmarkt von Juli 1968 bis Juni 1995 ( $t=1, \ldots, 324$ Monate)

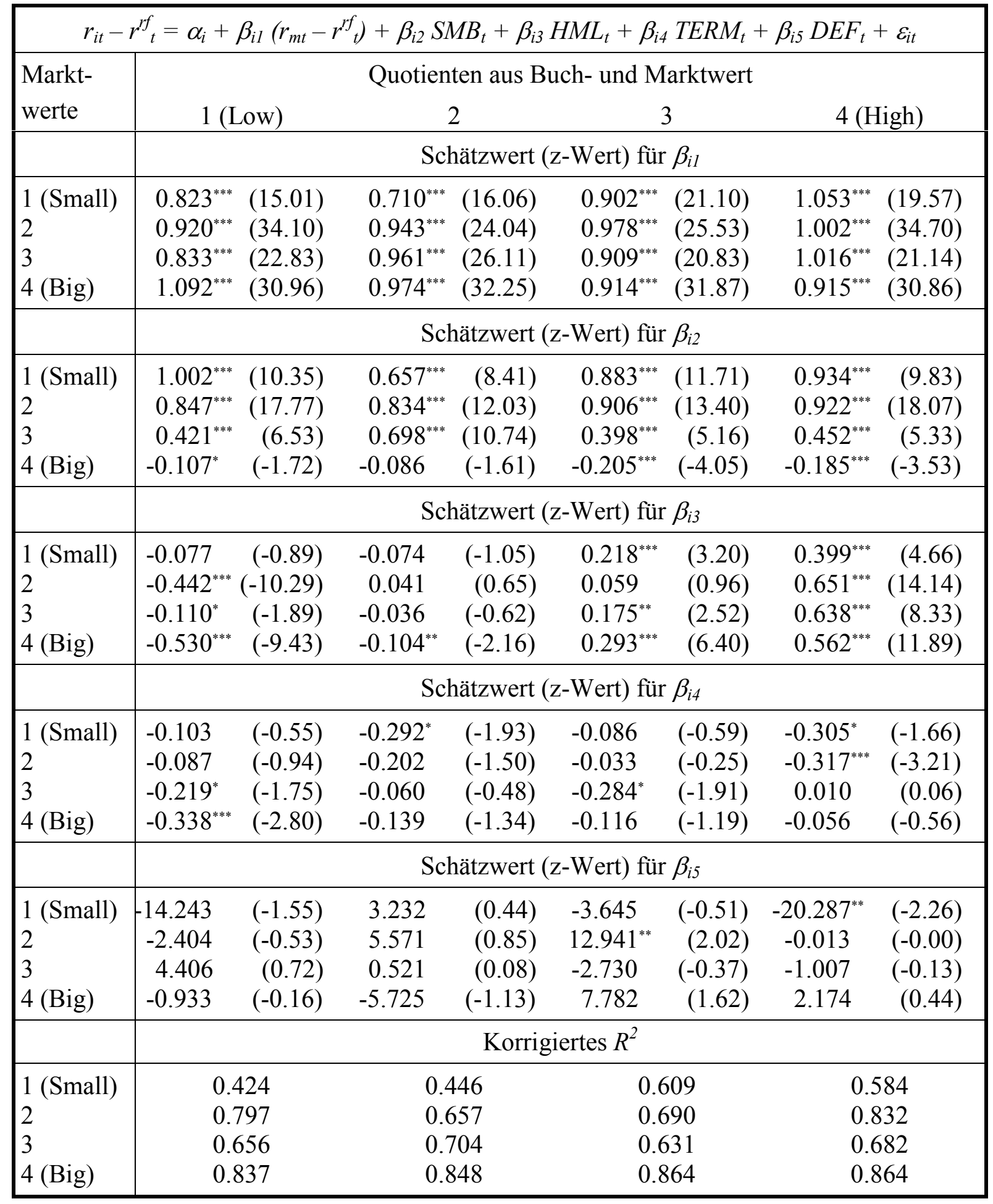

Anmerkungen:

*, ** bzw. *** bedeutet, dass nach dem zweiseitigen Parametertest auf den Wert Null die jeweilige erklärende Variable zu einem Signifikanzniveau von $10 \%, 5 \%$ bzw. $1 \%$ einen Einfluss auf $r_{i t}-r^{r f}{ }_{t}$ besitzt. 


\subsubsection{Analyse der Konstante $\alpha_{i}$}

Im Folgenden wird nun untersucht, inwieweit die durchschnittlichen Prämien der fünf betrachteten Risikofaktoren am deutschen Aktienmarkt Portfolio-Renditen im Querschnitt erklären können. Für diesen Fall sollte die Konstante $\alpha_{i}$ bei der Schätzung des CAPM oder der verschiedenen Multifaktormodelle nicht signifikant von Null verschieden sein. Eine wichtige Fragestellung ist dabei, ob das Dreifaktorenmodell, das offensichtlich ganz wesentlich die zeitliche Streuung von Aktienrenditen abbilden kann, auch in dieser Hinsicht einen hohen Erklärungsgehalt aufweist. In Tabelle 6 sind die entsprechenden Schätz- und z-Werte für $\alpha_{i}$ in den verschiedenen zuvor bereits betrachteten Zeitreihen-Regressionen abgebildet. Ein wesentliches Ergebnis der Tabelle sind die betragsmäßig äußerst geringen Schätzwerte mit der Folge, dass die Konstante nur selten signifikant von Null verschieden ist. Auffällig ist dabei, dass dieses Resultat prinzipiell für alle betrachteten Multifaktormodelle gilt. Aber selbst im CAPM ist $\alpha_{i}$ mit Schätzwerten zwischen - 0.003 (Portfolio 4-1) und 0.005 (Portfolio 2-4) lediglich bei sechs der 16 Aktienportfolios zu einem Signifikanzniveau von 10\% und davon ausschließlich bei Portfolio 2-4 zu einem Signifikanzniveau von 1\% von Null verschieden.

Bei der Schätzung des Zweifaktorenmodells, das die beiden Risikofaktoren $T E R M_{t}$ und $D E F_{t}$ des Anleihenmarktes enthält, ist die Konstante bei drei Aktienportfolios zu einem Signifikanzniveau von 5\% von Null verschieden. Bei Portfolio 1-4 zeigt sich in diesem Multifaktormodell über alle betrachteten Modelle mit 0.015 auch der betragsmäßig größte Schätzwert für $\alpha_{i}$. Bei der Schätzung des Zweifaktorenmodells, das die beiden Risikofaktoren $S M B_{t}$ und $H M L_{t}$ des Aktienmarktes enthält, ist die Konstante lediglich bei Portfolio 4-4 zu einem Signifikanzniveau von $10 \%$ von Null verschieden. Insbesondere ergibt sich aber im Dreifaktorenmodell, dass die Konstante bei allen Aktienportfolios nicht signifikant von Null verschieden ist. Die betragsmäßig größten Schätz- und z-Werte lauten hier 0.001 und 1.31 (bei Portfolio 4-4). Dementsprechend kann das Dreifaktorenmodell, das am deutschen Aktienmarkt die zeitliche Streuung von Renditen gut abbildet, bei der Betrachtung der Schätz- und z-Werte für $\alpha_{i}$ auch Portfolio-Renditen im Querschnitt sehr gut erklären. Allerdings sollte dabei berücksichtigt werden, dass auch das CAPM und die Zweifaktorenmodelle, insbesondere bei der Einbeziehung von $S M B_{t}$ und $H M L_{t}$, sowie das Fünffaktorenmodell einen relativ hohen Erklärungsgehalt für Aktienportfolio-Renditen im Querschnitt aufweisen. Die häufig im Fünffaktorenmodell gegenüber dem Dreifaktorenmodell bei den 16 Aktienportfolios betragsmäßig etwas höheren Schätz- und z-Werte für $\alpha_{i}$ mit der Folge, dass die Konstante bei Portfolio 2-3 
und Portfolio 1-4 zu einem Signifikanzniveau von 10\% und 5\% von Null verschieden ist, sind alleine der Einbeziehung von $D E F_{t}$ zuzuschreiben. Im Vierfaktorenmodell ${ }^{30}$ ohne Einbeziehung von $D E F_{t}$ ergeben sich nämlich nahezu identische Schätz- und z-Werte für $\alpha_{i}$ wie im Dreifaktorenmodell.

Allerdings unterscheiden sich diese Resultate am deutschen Aktienmarkt partiell deutlich von denjenigen am US-amerikanischen oder britischen Aktienmarkt. ${ }^{31}$ An diesen beiden Aktienmärkten ergeben sich im CAPM bei den jeweils 25 betrachteten Aktienportfolios starke Variationen (für die USA zwischen -0.22 und 0.57 und für Großbritannien zwischen -0.24 und 1.22) der Schätzwerte für $\alpha_{i}$, so dass die Konstante teilweise zu einem Signifikanzniveau von $1 \%$ und relativ häufig zu einem Signifikanzniveau von $10 \%$ von Null verschieden ist. Darüber hinaus steigen die Schätzwerte systematisch mit wachsendem Quotienten aus Buch- und Marktwert sowie (außer bei einem geringen Quotienten aus Buch- und Marktwert für die USA) mit sinkendem Marktwert in den Aktienportfolios an. Am US-amerikanischen Aktienmarkt ist ein solches Verhalten auch im Zweifaktorenmodell, das die beiden Risikofaktoren $T E R M_{t}$ und $D E F_{t}$ des Anleihenmarktes einbezieht, zu erkennen. Offensichtlich kann also der Einfluss des Marktwertes sowie des Quotienten aus Buch- und Marktwert auf die Aktienrenditen durch dieses Zweifaktorenmodell und insbesondere durch das CAPM nicht absorbiert werden. Dieses Verhalten bei der Schätzung des CAPM und des Zweifaktorenmodells ist auch am deutschen Aktienmarkt zu beobachten, allerdings in extrem abgeschwächter Form. Für Großbritannien zeigt sich diese Entwicklung im Gegensatz zu den USA und zur BRD bei Schätzwerten zwischen -0.32 und 0.78 sogar im Dreifaktorenmodell. Dabei ist die Konstante für Großbritannien in ähnlicher Häufigkeit wie im CAPM signifikant von Null verschieden, so dass das Dreifaktorenmodell am britischen Aktienmarkt Portfolio-Renditen im Querschnitt offensichtlich nicht befriedigend erklären kann.

Am US-amerikanischen Aktienmarkt ergibt sich wie am deutschen Aktienmarkt auch im Zweifaktorenmodell, das die beiden Risikofaktoren $S M B_{t}$ und $H M L_{t}$ des Aktienmarktes enthält, und im Fünffaktorenmodell kein systematischer Einfluss des Marktwertes sowie des Quotienten aus Buch- und Marktwert in den Portfolios auf die Schätzwerte für $\alpha_{i}$. Im Gegensatz zur BRD sind für die USA die Schätz- und z-Werte für $\alpha_{i}$ im Dreifaktoren- und Fünf-

${ }^{30}$ Die entsprechenden Schätzergebnisse sind in diesem Papier nicht gesondert ausgewiesen.

${ }^{31} \mathrm{Zu}$ beachten ist, dass die Schätz- und z-Werte für $\alpha_{i}$ in der Studie von Berkowitz/Qiu (2001) für den kanadischen Aktienmarkt nicht ausgewiesen und in der Studie von Hussain et al. (2002) für den britischen Aktienmarkt lediglich das CAPM und das Dreifaktorenmodell analysiert werden. 
faktorenmodell nahezu identisch. Vor allem aber unterscheidet sich an diesen beiden Aktienmärkten in allen Multifaktormodellen vehement das Niveau der Schätz- und z-Werte. So variieren die Schätzwerte für die USA in diesem Zweifaktorenmodell zwischen 0.24 und 0.79 und im Dreifaktorenmodell zwischen -0.34 und 0.21 , so dass die Konstante im Zweifaktorenmodell bei acht der 25 untersuchten Aktienportfolios und im Dreifaktorenmodell (wie auch im Fünffaktorenmodell) bei immerhin drei Portfolios zu einem Signifikanzniveau von 1\% von Null verschieden ist. Festzuhalten ist damit, dass das CAPM und alle Multifaktormodelle, insbesondere aber das Dreifaktorenmodell bei der Betrachtung der Schätz- und z-Werte für $\alpha_{i}$ Portfolio-Renditen im Querschnitt am deutschen Aktienmarkt wesentlich besser erklären können als am US-amerikanischen Aktienmarkt. 
Tabelle 6: Schätzwerte (z-Werte) für die Konstante $\alpha_{i}$ in den verschiedenen linearen Regressionsmodellen: Deutscher Aktienmarkt von Juli 1968 bis Juni 1995 ( $t=1, \ldots, 324$ Monate)

\begin{tabular}{|c|c|c|c|c|c|c|c|c|}
\hline \multirow{2}{*}{$\begin{array}{l}\text { Markt- } \\
\text { werte }\end{array}$} & \multicolumn{8}{|c|}{ Quotienten aus Buch- und Marktwert } \\
\hline & \multicolumn{2}{|c|}{1 (Low) } & \multicolumn{2}{|c|}{2} & \multicolumn{2}{|c|}{3} & \multicolumn{2}{|c|}{4 (High) } \\
\hline \multicolumn{9}{|c|}{ Modell: $r_{i t}-r_{t}^{r f}=\alpha_{i}+\beta_{i l}\left(r_{m t}-r_{t}^{r f}\right)+\varepsilon_{i t}$} \\
\hline 1 (Small) & 0.001 & $(0.42)$ & -0.000 & $(-0.06)$ & 0.002 & $(0.88)$ & $0.004^{*}$ & $(1.68)$ \\
\hline 2 & 0.001 & $(0.36)$ & 0.001 & $(0.30)$ & 0.001 & $(0.78)$ & $0.005^{* * *}$ & $(2.73)$ \\
\hline & -0.000 & $(-0.15)$ & 0.001 & $(0.46)$ & 0.000 & $(0.20)$ & $0.003^{*}$ & $(1.67)$ \\
\hline 4 (Big) & $-0.003^{*}$ & $(-1.92)$ & $-0.002^{*}$ & $(-1.65)$ & 0.000 & $(0.22)$ & $0.003^{* *}$ & $(2.54)$ \\
\hline \multicolumn{9}{|c|}{ Modell: $r_{i t}-r_{t}^{r f}=\alpha_{i}+\beta_{i 2} S M B_{t}+\beta_{i 3} H M L_{t}+\varepsilon_{i t}$} \\
\hline 1 (Small) & 0.002 & $(0.66)$ & 0.001 & $(0.43)$ & 0.002 & $(0.70)$ & 0.004 & $(1.20)$ \\
\hline & 0.003 & $(1.50)$ & 0.001 & $(0.60)$ & 0.002 & $(0.90)$ & 0.003 & $(1.44)$ \\
\hline & 0.002 & $(0.78)$ & 0.002 & $(0.93)$ & 0.001 & $(0.56)$ & 0.003 & $(1.03)$ \\
\hline 4 (Big) & 0.002 & $(0.88)$ & 0.001 & $(0.57)$ & 0.002 & $(0.88)$ & $0.004^{*}$ & (1.76) \\
\hline \multicolumn{9}{|c|}{ Modell: $r_{i t}-r_{t}^{r f}=\alpha_{i}+\beta_{i 4} T E R M_{t}+\beta_{i 5} D E F_{t}+\varepsilon_{i t}$} \\
\hline 1 (Small) & $0.009^{* *}$ & $(2.00)$ & 0.002 & $(0.54)$ & 0.007 & $(1.62)$ & $0.015^{* * *}$ & $(2.92)$ \\
\hline & 0.005 & (1.35) & 0.003 & $(0.71)$ & 0.002 & $(0.38)$ & $0.010^{* *}$ & $(2.20)$ \\
\hline & 0.002 & $(0.52)$ & 0.005 & (1.13) & 0.005 & (1.19) & 0.009 & (1.61) \\
\hline 4 (Big) & 0.002 & $(0.35)$ & 0.004 & $(0.86)$ & 0.002 & $(0.40)$ & 0.007 & $(1.40)$ \\
\hline \multicolumn{9}{|c|}{ Modell: $r_{i t}-r_{t}^{r f}=\alpha_{i}+\beta_{i 1}\left(r_{m t}-r_{t}^{r f}\right)+\beta_{i 2} S M B_{t}+\beta_{i 3} H M L_{t}+\varepsilon_{i t}$} \\
\hline 1 (Small) & -0.000 & $(-0.22)$ & -0.001 & $(-0.57)$ & -0.001 & $(-0.44)$ & 0.001 & $(0.37)$ \\
\hline 2 & 0.001 & $(0.80)$ & -0.001 & $(-0.72)$ & -0.000 & $(-0.28)$ & 0.001 & $(0.65)$ \\
\hline & -0.001 & $(-0.37)$ & -0.000 & $(-0.24)$ & -0.001 & $(-0.64)$ & 0.000 & $(0.06)$ \\
\hline 4 (Big) & -0.001 & $(-0.45)$ & -0.001 & $(-1.16)$ & -0.001 & $(-0.50)$ & 0.001 & $(1.31)$ \\
\hline \multicolumn{9}{|c|}{ Modell: $r_{i t}-r^{r f}{ }_{t}=\alpha_{i}+\beta_{i 1}\left(r_{m t}-r^{r f}{ }_{t}\right)+\beta_{i 2} S M B_{t}+\beta_{i 3} H M L_{t}+\beta_{i 4} T E R M_{t}+\beta_{i 5} D E F_{t}+\varepsilon_{i t}$} \\
\hline 1 (Small) & 0.004 & (1.17) & -0.002 & $(-0.65)$ & 0.001 & $(0.18)$ & $0.007^{* *}$ & $(2.11)$ \\
\hline 2 & 0.002 & $(0.92)$ & -0.003 & $(-1.08)$ & $-0.005^{*}$ & $(-1.82)$ & 0.001 & $(0.45)$ \\
\hline & -0.002 & $(-0.77)$ & -0.000 & $(-0.20)$ & -0.000 & $(-0.02)$ & 0.000 & $(0.14)$ \\
\hline 4 (Big) & -0.000 & $(-0.07)$ & 0.001 & $(0.30)$ & -0.003 & $(-1.60)$ & 0.001 & $(0.40)$ \\
\hline
\end{tabular}

Anmerkungen:

*, ** bzw. *** bedeutet, dass die Nullhypothese $H_{0}: \alpha_{i}=0$ zu einem Signifikanzniveau von 10\%, 5\% bzw. 1\% abgelehnt werden kann.

\section{Zusammenfassung und Schlussfolgerungen}

Dieses Papier untersucht, inwieweit Multifaktormodelle nach Fama/French (1993) am deutschen Aktienmarkt die zeitliche Streuung von Renditen abbilden und die Renditen von Portfolios (sortiert auf der Grundlage des Marktwertes sowie des Quotienten aus Buch- und Marktwert) im Querschnitt erklären können. Bei den linearen Zeitreihen-Regressionen im 
CAPM und in verschiedenen Multifaktormodellen ergibt sich, dass ein Dreifaktorenmodell, das neben der Überschussrendite des Aktienmarktes über den risikolosen Zins zwei weitere Risikofaktoren des Aktienmarktes (die sich aus dem Marktwert sowie dem Quotienten aus Buch- und Marktwert ableiten) enthält, eine deutlich höhere Erklärungskraft besitzt als das traditionelle CAPM. Dagegen weisen zwei Risikofaktoren des Anleihenmarktes (die sich aus der Zinsstruktur und dem Ausfallrisiko ableiten) in einem Fünffaktorenmodell keinen zusätzlichen Erklärungsgehalt für die Überschussrendite der Aktienportfolios auf. Gegenüber dem US-amerikanischen und kanadischen Aktienmarkt kann allerdings das Dreifaktorenmodell am deutschen Aktienmarkt die zeitliche Streuung von Renditen nur schlechter abbilden. Dagegen werden für die BRD Aktienportfolio-Renditen im Querschnitt wesentlich besser erklärt als für die USA. Am deutschen Aktienmarkt weisen in dieser Hinsicht (teilweise im Gegensatz zum US-amerikanischen und britischen Aktienmarkt) nicht nur das Dreifaktorenmodell, sondern auch die anderen betrachteten Multifaktormodelle und das CAPM einen hohen Erklärungsgehalt auf.

Bemerkenswert sind am deutschen Aktienmarkt die Resultate für den Risikofaktor des Aktienmarktes, der sich aus dem Marktwert ableitet. Sowohl im Zweifaktoren- als auch im Dreifaktoren- und Fünffaktorenmodell besitzt $S M B_{t}$ entsprechend den Zeitreihen-Regressionen eine große Bedeutung zur Erklärung der Überschussrendite der betrachteten Aktienportfolios. Demgegenüber kann entsprechend der deskriptiven Analyse für die BRD (im Gegensatz zu den USA, Kanada und Großbritannien) sowohl in Bezug auf die Höhe von $S M B_{t}$ als auch in Bezug auf die durchschnittlichen monatlichen Aktienrenditen keine systematische Relation zwischen der Aktienrendite und dem Marktwert festgestellt werden. Dagegen ergibt sich am deutschen Aktienmarkt (ähnlich wie am US-amerikanischen, kanadischen und britischen Aktienmarkt) zwischen dem Quotienten aus Buch- und Markwert und der Aktienrendite eine positive Korrelation. Gleichzeitig besitzt auch der Risikofaktor $H M L_{t}$ des Aktienmarktes, der sich aus dem Quotienten aus Buch- und Marktwert ableitet, nach den Zeitreihen-Regressionen in allen betrachteten Multifaktorenmodellen eine große Bedeutung zur Erklärung der Überschussrendite der Aktienportfolios.

Der Einfluss der beiden Risikofaktoren des Anleihenmarktes auf die Überschussrendite der Aktienportfolios wird am deutschen Aktienmarkt (ebenso wie am US-amerikanischen und kanadischen Aktienmarkt) in einem Fünffaktorenmodell durch die drei Risikofaktoren des Aktienmarktes absorbiert. Auffällig sind dabei aber vor allem die unplausiblen Schätzergeb- 
nisse beim Risikofaktor $D E F_{t}$, der sich aus dem Ausfallrisiko ableitet. Sowohl im Zweifaktoren- als auch im Fünffaktorenmodell zeigen sich für die BRD bei allen betrachteten Aktienportfolios äußerst hohe geschätzte Standardabweichungen der geschätzten Parameter. Diese Schätzergebnisse dürften vor allem mit der Berechnung von $D E F_{t}$ durch Umlaufrenditen von Portfolios langfristiger Unternehmens- und Staatsanleihen zusammen hängen. In Zukunft sollte deshalb unter Verwendung detaillierter Daten ein verlässlicher Index für deutsche Unternehmensanleihen konstruiert und damit analog zu Fama/French (1993) für die USA der Faktor $D E F_{t}$ mit Hilfe der monatlichen Wertänderung eines Portfolios langfristiger Unternehmensanleihen berechnet werden. Interessant wäre dann die Untersuchung, ob sich bei der Schätzung der entsprechenden Multifaktormodelle für diesen Risikofaktor ähnliche Ergebnisse zeigen wie in diesem Papier.

Forschungsbedarf verbleibt auch dahingehend, inwieweit Multifaktormodelle und insbesondere das Dreifaktorenmodell am deutschen Aktienmarkt die Überschussrendite von Aktienportfolios erklären können, die nicht auf der Grundlage des Marktwertes sowie des Quotienten aus Buch- und Marktwert, sondern zum Beispiel auf der Grundlage des Kurs-Gewinn-Verhältnisses, der Cash-Flow-Kurs-Relation oder des Umsatzwachstums sortiert werden. Hintergrund einer solchen Betrachtung wäre die Untersuchung, ob die betrachteten Multifaktormodelle nicht nur Anomalien einfangen können, die auf dem Marktwert oder den Quotienten aus Buch- und Marktwert beruhen. Entsprechend den Ergebnissen von Hussain et al. (2002) können am britischen Aktienmarkt entgegen den Resultaten von Fama/French (1996) am USamerikanischen Aktienmarkt viele dieser Anomalien nicht durch das Dreifaktorenmodell aufgefangen werden. Nach unserer Kenntnis ist diese Fragestellung bisher noch nicht am deutschen Aktienmarkt analysiert worden.

Weitere zukünftige Analysen könnten ferner am deutschen Aktienmarkt ein auf Unternehmenscharakteristika basierendes Modell entsprechend Daniel/Titman (1997) und Daniel et al. (2001), die den US-amerikanischen und japanischen Aktienmarkt betrachten, untersuchen. Interessant wäre dabei insbesondere der Vergleich mit dem in diesem Papier betrachteten auf Risikofaktoren aufbauenden Modell von Fama/French (1993). Sinnvoll wäre am deutschen Aktienmarkt auch eine vertiefende Analyse der Risikofaktoren $S M B_{t}$ und $H M L_{t}$ des Aktienmarktes wie zum Beispiel die Analyse von Unternehmen mit negativem Buchwert entsprechend Hussain et al. (2002) am britischen Aktienmarkt. Auch die Untersuchung der zeitlichen Stabilität der Schätzergebnisse könnte weitere nützliche Einblicke in die Relevanz von Multi- 
faktormodellen und insbesondere des Dreifaktorenmodells entsprechend Fama/French (1993) im Allgemeinen und deren Gültigkeit am deutschen Aktienmarkt im Besonderen geben.

\section{Literatur}

Banz, Rolf W. (1981), The Relationship Between Return and Market Value of Common Stocks, in: Journal of Financial Economics, Vol. 9. S. 3-18.

Basu, Sanjoy (1983), The Relationship Between Earnings Yield, Market Value and Return for NYSE Common Stocks: Further Evidence, in: Journal of Financial Economics, Vol. 12. S. 129-156.

Berkowitz, Michael K., Qiu, Jiaping (2001), Common Risk Factors in Explaining Canadian Equity Returns, University of Toronto.

Bunke, Olaf, Sommerfeld, Volker, Stehle, Richard (1998), Semiparametric Modeling of the Cross-Section of Expected Returns in the German Stock Market, Humboldt-Universität zu Berlin.

Daniel, Kent, Titman, Sheridan (1997), Evidence on the Characteristics of Cross Sectional Variation in Stock Returns, in: The Journal of Finance, Vol. 52, S. 1-33.

Daniel, Kent, Titman, Sheridan, Wei, John K. C. (2001), Explaining the Cross-Section of Stock Return in Japan: Factors or Characteristics?, in: The Journal of Finance, Vol. 56, S. 743-766.

DeBondt, Werner F. M., Thaler, Richard (1985), Does the Stock Market Overreact?, in: The Journal of Finance, Vol. 40. S. 793-805.

Davis, James L., Fama, Eugene F., French, Kenneth R. (2000), Characteristics, Covariances and Average Returns: 1929 to 1997, in: The Journal of Finance, Vol. 55. S. 389-406. 
Fama, Eugene F., French, Kenneth R. (1992), The Cross-Section of Expected Stock Returns, in: The Journal of Finance, Vol. 47. S. 427-465.

Fama, Eugene F., French, Kenneth R. (1993), Common Risk Factors in the Returns on Stocks and Bonds, in: Journal of Financial Economics, Vol. 33. S. 3-56.

Fama, Eugene F., French, Kenneth R. (1995), Size and Book-to-Market Factors in Earnings and Returns, in: The Journal of Finance, Vol. 50. S. 131-155.

Fama, Eugene F., French, Kenneth R. (1996), Multifactor Explanations of Asset Prising Anomalies, in: The Journal of Finance, Vol. 51. S. 55-84.

Fama, Eugene F., French, Kenneth R. (1998), Value Versus Growth: The International Evidence, in: The Journal of Finance, Vol. 53. S. 1975-1999.

Fama, Eugene F., MacBeth, James D. (1973), Risk, Return, and Equilibrium: Empirical Tests, in: Journal of Political Economy, Vol. 81. S. 607-636.

Hussain, Iki, Toms, Steve, Diacon, Stephen (2002), Financial Distress, Single and Multifactor Tests and Comparisons of Asset Pricing Anomalies: New Evidence, Nottingham University Business School.

Lintner, John (1965), The Valuation of Risk Assets and the Selection of Risky Investments in Stock Portfolios and Capital Budgets, in: Review of Economics and Statistics, Vol. 47. S. 1337.

Merton, Robert C. (1973), An Intertemporal Capital Asset Pricing Model, in: Econometrica, Vol. 41, S. 867-887.

Rosenberg, Barr, Reid, Kenneth, Lanstein, Ronald (1985), Persuasive Evidence of Market Inefficiency, in: Journal of Portfolio Management, Vol. 9. S. 18-28.

Sattler, Ralph R. (1994), Renditeanomalien am deutschen Aktienmarkt, Aachen. 
Schulz, Anja, Stehle, Richard (2002), Buchwert-Marktwert-Verhältnis, Size und Beta als Erklärungsvariable für die Renditen deutscher Aktien, Humboldt-Universität zu Berlin.

Sharpe, William F. (1964), Capital Asset Prices: A Theory of Market Equilibrium under Conditions of Risk, in: The Journal of Finance, Vol. 19. S. 425-442.

Stehle, Richard (1997), Der Size-Effekt am deutschen Aktienmarkt, in: Zeitschrift für Bankrecht und Bankwirtschaft, Vol. 9. S. 237-260.

Stehle, Richard, Hartmond, Anette (1991), Durchschnittsrenditen deutscher Aktien 19541988, in: Kredit und Kapital, Vol. 24. S. 371-411.

Stock, Detlev (2002), Zur Relevanz von CAPM-Anomalien für den deutschen Aktienmarkt, Frankfurt am Main.

Wallmeier, Martin (2000), Determinanten erwarteter Renditen am deutschen Aktienmarkt Eine empirische Untersuchung anhand ausgewählter Kennzahlen, in: ZfbF, Vol. 52. S. 27-57. 